\title{
Analysis of Internally Bandlimited Multistage Cubic-Term Generators for RF Receivers
}

\author{
Edward A. Keehr, Student Member, IEEE, and Ali Hajimiri, Member, IEEE
}

\begin{abstract}
Adaptive feedforward error cancellation applied to correct distortion arising from third-order nonlinearities in RF receivers requires low-noise low-power reference cubic nonidealities. Multistage cubic-term generators utilizing cascaded nonlinear operations are ideal in this regard, but the frequency response of the interstage circuitry can introduce errors into the cubing operation. In this paper, an overview of the use of cubic-term generators in receivers relative to other applications is presented. An interstage frequency response plan is presented for a receiver cubic-term generator and is shown to function for arbitrary three-signal third-order intermodulation generation. The noise of such circuits is also considered and is shown to depend on the total incoming signal power across a particular frequency band. Finally, the effects of the interstage group delay are quantified in the context of a relevant communication standard requirement.
\end{abstract}

Index Terms-Adaptive signal processing, digital linearization, mixed-signal linearization, postdistortion, RF receivers, system-level linearization, wireless communications.

\section{INTRODUCTION}

A DAPTIVE feedforward error cancellation in RF receivers seeks to eliminate self-generated interference arising due to the interaction of desired and undesired signals with circuit nonidealities within the receiver. In order to accomplish this, as shown in Fig. 1, the feedforward loops employ rough models of the circuit nonidealities to recreate approximations to the interference signals. Adaptive filtering is used in order to reconcile any baseband linear time invariant differences between the original interference signals and the reference interference signals prior to cancellation. For example, in Fig. 1, the simple case of a single-tap LMS filter is used to compensate for an unknown gain difference between the main receiver path and the auxiliary feedforward loops.

In recent years, this concept has been proposed for use in RF receivers to remove second-order intermodulation (IM2) distortion and dc offset [1], third-order intermodulation (IM3) distortion [2], [3], and signal interference resulting from in-phase and quadrature (I-Q) mismatch in IF receiver circuitry [4], [5] and was described as a general concept in [5]. Other examples of the use of adaptive signal processing in receivers include the cancellation of error-producing signals themselves [6] and additional

Manuscript received April 07, 2008; revised August 08, 2008. First published October 31, 2008; current version published August 21, 2009. This work was supported in part by the Lee Center for Advanced Networking and in part by a National Defense Science and Engineering Graduate Fellowship. This paper was recommended by Associate Editor E. A. M. Klumperink.

E. A. Keehr is with the California Institute of Technology, Pasadena, CA 91125 USA (e-mail: keehr@caltech.edu).

A. Hajimiri is with the Microelectronics Laboratory, California Institute of Technology, Pasadena, CA 91125 USA (e-mail: hajimiri@ caltech.edu).

Digital Object Identifier 10.1109/TCSI.2008.2008282

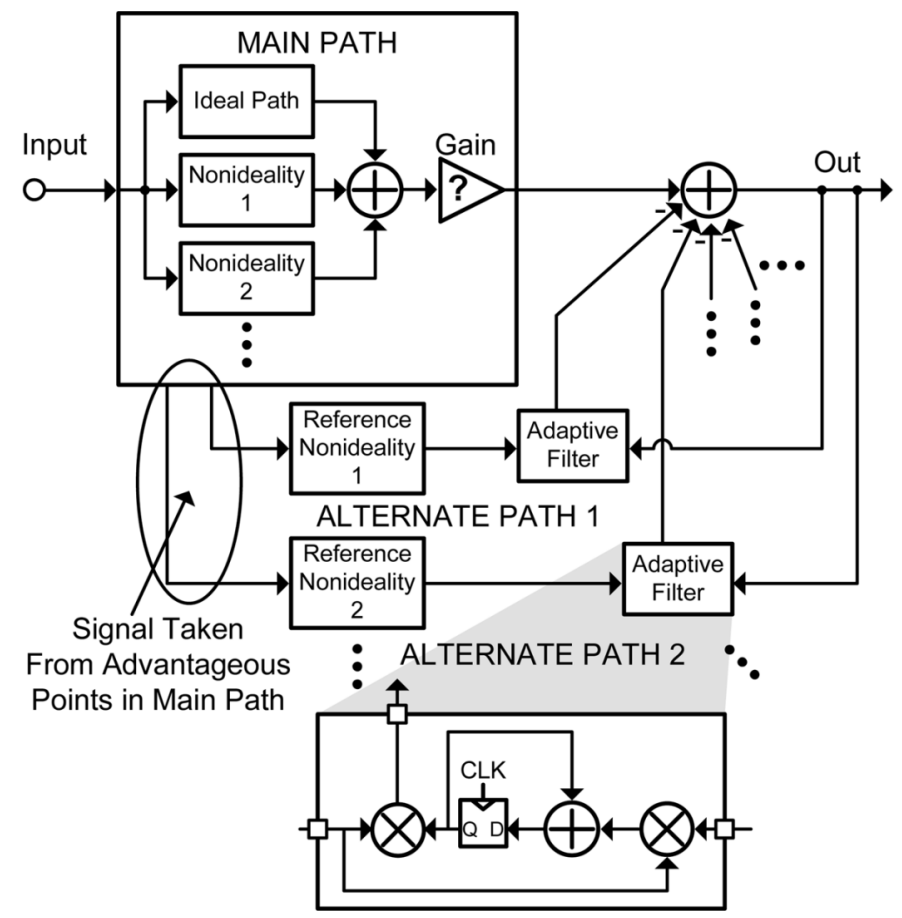

Fig. 1. General adaptive feedforward error-cancellation concept.

techniques to remove the effects of dc offset and I-Q mismatch in direct-conversion receivers [7], [8].

Because the error-cancellation schemes in [2] and [3] generate the reference IM3 distortion in the digital domain, the analog-to-digital converters (ADCs) in these systems are required to accurately digitize all IM3-producing signals. In many applications, such as in commercial Universal Mobile Telecommunications System (UMTS) receivers, the range of frequencies over which IM3-producing signals may occur is well over an order of magnitude greater than the bandwidth of the desired signal, substantially increasing the required area and power dissipation of the ADCs beyond what the original receiver required.

To overcome this limitation, the scheme shown in Fig. 2 was recently proposed [9], in which IM3 products are generated at analog RF by a CMOS cubic-term generator circuit. This scheme was shown to dramatically improve the receiver third-order intercept point (IIP3) at the frequencies of the large out-of-band blocking signals which dictate the receiver linearity requirements for frequency-division duplexing (FDD) UMTS (Region 1). These out-of-band blocking signals attain their peak voltage magnitude immediately after the LNA. Placing the cubic-term generator at this point then guarantees that the output reference IM3 products will be as large as possible for a given cubic-term generator topology, reducing the noise requirements of subsequent circuitry. 


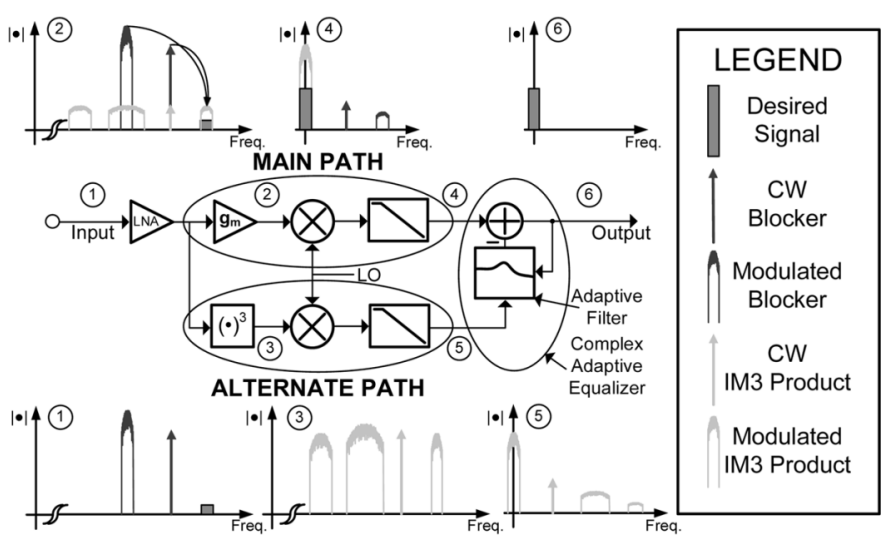

Fig. 2. Adaptive feedforward error-cancellation concept applied to RF receiver third-order distortion in [9].

In order to downconvert the reference IM3 products to baseband, a complete direct-conversion receiver is inserted between the cubic-term generator and the adaptive equalizer. Choosing the local-oscillator $(\mathrm{LO})$ frequency in the alternate receiver path to be the same as that in the original main receiver guarantees that the proper set of IM3 products are centered around DC at baseband, while the other IM3 products are attenuated to negligible levels by baseband filtering.

\section{Prior Art in Cubic-Term Generators}

Cubic-term generators have been extensively used in the past for the predistortion of nonlinear wireline, radio, and laser power amplifiers (PAs). Many early predistortion circuits utilized the nonlinear impedance characteristic of back-to-back diodes [10]-[12], while an active implementation of such a circuit with higher cubing gain using bipolar junction transistor devices was reported in [13]. Such architectures were not considered for the project in [9] as they are not easily implemented in CMOS processes. CMOS cubic-term generators that utilize the third-order Taylor series coefficients of the MOSFET have also been presented [14]-[16]. However, as the MOSFET is a square-law device, the third-order Taylor series coefficient is relatively weak. Furthermore, the cubing operation heavily attenuates the IM3 output signal with respect to the noise of the IM3-producing devices. As will be discussed in Section V, the noise of the cubic-term generator is of prime importance in the receiver of [9] and renders the use of these architectures unattractive in such an application, although they may represent competitive design options when noise is not an issue.

Cubic-term generators that realize polynomial function generation by using a cascade of multiplier operations represent a superior approach to cubic-term generator design in this regard. Predistortion circuits using cascaded Gilbert cell multipliers have been implemented with discrete components [17] and in BiCMOS [18] and CMOS [19] processes. Nielsen et al. [20] propose the use of multipliers modified from [21] that are based on a sum-and-difference squaring technique which utilizes the square-law dependence of the MOS transconductance but have not reported on a complete predistortion circuit to the knowledge of the authors of this paper. Architectures of this class potentially reap a noise benefit relative to those using explicit third-order nonlinearities, as the initial nonlinear products

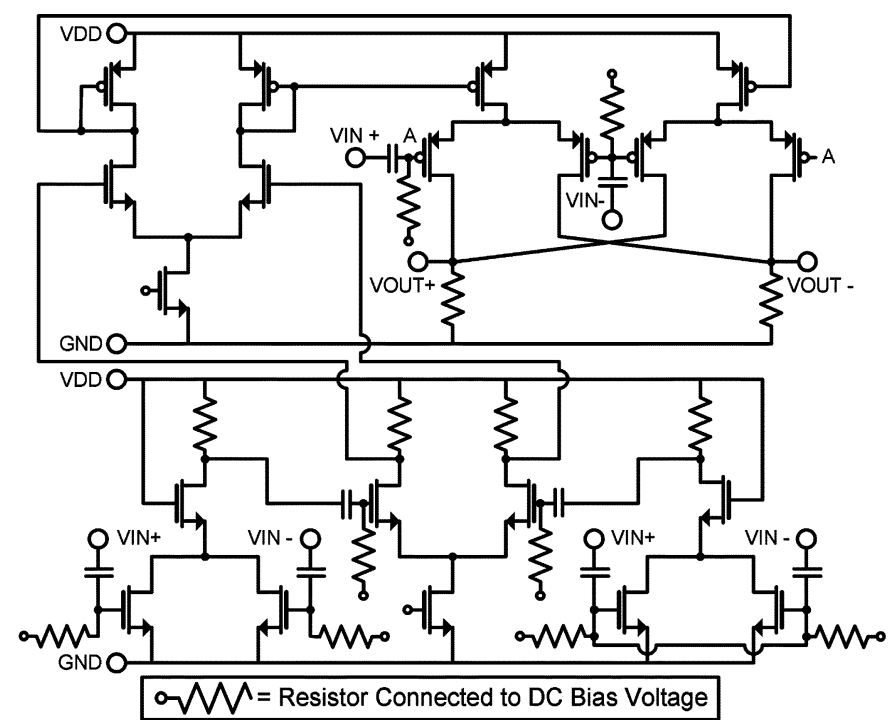

Fig. 3. Schematic of the cubic-term generator utilized in [9].

are only attenuated with respect to the noise of the nonlinear devices as the square of the input signal, not the cube.

\section{Proposed Cubic-Term Generator}

The cubic-term generator shown in Fig. 3 was implemented for the receiver in [9] and will be the focus of this paper. Like those of [17]-[19], it utilizes multiple nonlinear operations to generate third-order distortion, and hence, much of the analysis in this paper applies to these architectures as well. The first nonlinear operation in this case is performed by the simple MOS squaring transconductor in the lower left-hand corner of the schematic. This choice is made in order to avoid the generation of higher order intermodulation products associated with the nonlinearity of the Gilbert cell current-commutating devices. It also represents a simpler approach than that described in [21], using fewer noise-producing transistors (2 versus 12 ).

As the MOS squaring circuit produces a single-ended output, it must be followed by an active balun to recast the signal differentially. One potential issue with this scheme is that the squaring circuit directly passes the common-mode signal. If the balun negative terminal were grounded, the common-mode signal would be recast differentially as well and would propagate through the remainder of the circuit. In order to provide some measure of common-mode rejection, a dummy squaring circuit is added to the negative terminal of the balun. With the gate terminals of the dummy squaring circuit tied together, this circuit only passes the common-mode signal. Hence, the common-mode signal is rejected by the common-mode-rejection ratio of the balun and subsequent gain circuits. The use of a balun does not constitute an extra burden on the circuit as it also functions as a gain stage to suppress the effective noise contributions of subsequent circuitry.

The final multiplication of the cubic-term generator is performed by a Gilbert cell multiplier. In this case, the nonlinearity of the current-commutating devices can be improved at the expense of gain by increasing the multiplying device overdrive voltages or by attenuating the RF signal at this point via capacitive division. The lost gain can then be made up earlier in the circuit. The circuit, as implemented in [9], is somewhat 


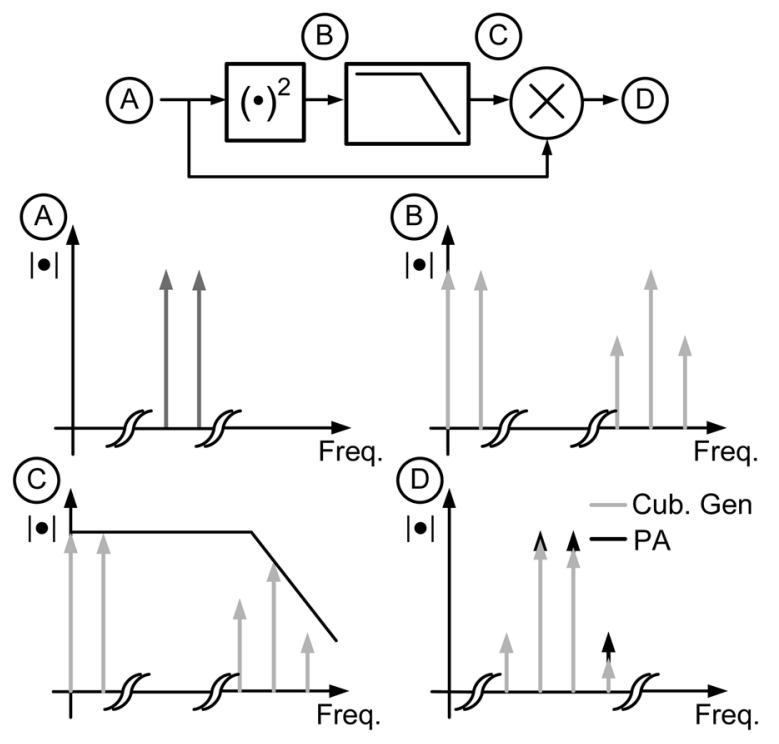

Fig. 4. Asymmetry of IM3 products due to memory effects of bandwidth limitation.

power-inefficient due to the voltage output at RF. This was done for testing purposes, but in a commercial implementation, the IM3 reference signal would be passed to a mixer switching pair in the current domain.

\section{REQUIREMENTS FOR PREDISTORTER CUBIC-TERM GENERATOR}

One of the chief requirements of the predistorter cubic-term generator is the maximum modulation bandwidth achievable for a given level of IM product suppression. Memory effects in the predistorted PA system, including nonconstant frequency response over the signal bands of interest, can result in asymmetric upper and lower IM3 sidebands, limiting the achievable error cancellation [14], [18], as shown in Fig. 4. For this reason, the design of effectively memoryless predistortion circuits is targeted [18], [19] so as not to contribute to this effect. Although a memory can be designed into the predistorter to compensate this effect, it is found that this technique is sensitive to frequency response deviations, and hence, it is desirable to keep the predistorter memoryless and to use other techniques to remove memory effects from the PA [22].

Another important requirement in systems only correcting for third-order nonlinearities is to minimize the ratio of fifth-order intermodulation (IM5) to IM3 products [10], [16], although the authors of [17] have shown that even an ideal cubic predistorter affects the IM5 products at the output of the amplifier and limits the performance of the system in their work. Cubing gain has also been mentioned as important in order for the predistorter to more easily match the amplitudes of the IM3 products in the PA [13], [16].

\section{REQUIREMENTS FOR RECEIVER CUBIC-TERM GENERATOR}

\section{A. Predistorter Requirements Not Vital in Receiver}

Memory effects limit the performance of predistortion linearization schemes because, if adaptive control is included at all, only the relative amplitude and phase of the compensatory IM products can be controlled. In the receiver, however, if some bandpass frequency response mismatch exists between the main and alternate paths, the complex adaptive equalizer can compensate for such frequency-dependent effects at baseband if more than one delayed filter tap is used.

Another important distinction in the case of the receiver cubic-term generator is that only one IM3 sideband falling around the receiver $\mathrm{LO}$ frequency $f_{\mathrm{LO}}$ is required in an out-of-band blocking scenario. Hence, the broadband asymmetry problem in Fig. 4 does not apply here. For the UMTS FDD specification [22], the required double-sided signal bandwidth to be retained is $3.84 \mathrm{MHz}$. It can be safely assumed that, based on the achieved linearization bandwidth results of [10], [11], [13], [14], and [16]-[18], maintaining a relatively constant frequency response around this frequency range is, in general, not difficult and that only the minimal frequency response correction is required of the adaptive equalizer.

Cubing gain is likewise not a vital parameter in that the RF receiver chain used in the alternate path itself typically has substantial gain after the downconversion to baseband.

\section{B. Vital Requirements of Receiver Cubic-Term Generator}

The requirements discussed in [10]-[19] are notable in that noise is not considered as a relevant design parameter. This is because the large signals processed by the predistorter are, in fact, desired signals and are well above the noise floor. This is not the case in the system presented in [9], in which the IM3-producing signals are undesired and the desired signal is buried underneath IM3 products over an order of magnitude greater. In this case, any noise present in the cubic-term generator, even if it is an order of magnitude less than the IM3 products, can add significantly to the noise floor of the receiver when the alternate path is enabled. Likewise, higher order distortion terms should be taken into account. Although they are typically much smaller than the IM3 products, they may still render the performance of the enhanced receiver inadequate to meet specifications. The linear term feedthrough of the desired signal must also be suppressed, as the desired signal at the reference input of the equalizer will be treated as an error by the adaptive algorithm. As the algorithm functions so as to minimize the mean squared error, it will attempt to strike a balance between eliminating the IM3 products and the desired signal, reducing small signal gain, IM3 cancellation, or both.

The relationship between signal and noise is typically quantified using an signal-to-noise ratio (SNR) metric. Since IM3 products are not the desired signals, we denote the ratio of these terms to the in-band noise as interference-to-noise ratio (INR). Generalizing this metric to other forms of error, including noise and other distortion terms, results in a quantity termed interference-to-error ratio (IER). Quantifying the performance impact of the alternate path on the main path receiver is easily done using these metrics. Taking the output of the alternate path as the output of the adaptive filter in Fig. 2, the adaptive equalizer enforces the equality of the IM3 products in the main and alternate paths (1), with any discrepancy in this equality counting toward the IER of the alternate path

$$
I_{\mathrm{MAIN}}=I_{\mathrm{ALT}} \text {. }
$$

After the cancellation of the IM3 products, there exist a residual error due to other IM products and a host of other 


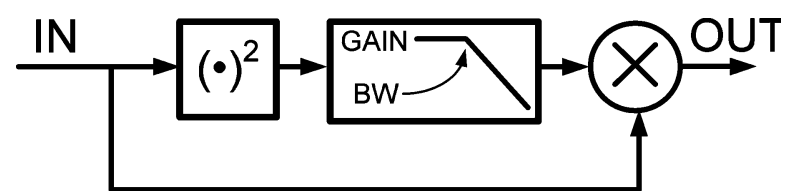

Fig. 5. Implementation of gain between nonlinear operations in multistage cubic-term generator.

effects. As depicted in (2), some of this error is due to the main path, and some is due to the alternate path. It is assumed in (2) that this error is uncorrelated to a good approximation

$$
E_{\mathrm{TOT}}^{2}=E_{\mathrm{MAIN}}^{2}+E_{\mathrm{ALT}}^{2} .
$$

Using (1) and (2), the following relationship can be derived:

$$
E_{\mathrm{TOT}}^{2}=E_{\mathrm{MAIN}}^{2}\left(1+\left(\frac{I E R_{\mathrm{MAIN}}}{I E R_{\mathrm{ALT}}}\right)^{2}\right) .
$$

The usefulness of (3) is that it can be used to set the error requirements on the alternate path circuitry, as the main path quantities and total allowed receiver error are typically known $a$ priori. Another relevant aspect of (3) is that, as the power of the IM3-producing blocker signals are varied, the IER quantities of the two paths roughly track, assuming that the third-order distortion products are by far the dominant ones in both paths, an assumption which is borne out in [9]. Therefore, if the input-referred error requirements are met under the peak, or worst case, blocking condition, when the input-referred error is highest, then the receiver will meet input-referred error requirements under all blocking conditions. Hence, the performance of the alternate path circuitry, including the cubic-term generator, is specified at this point. To put this quantity in perspective, it was shown that, in [9], for a UMTS receiver with uncorrected $I I P 3=-9 \mathrm{dBm}$, a reasonable error budgeting procedure calls for $I E R_{\mathrm{ALT}}>31 \mathrm{~dB}$ and $I E R_{\mathrm{CUB}}>34 \mathrm{~dB}$ under peak blocking.

\section{INTERSTAGE BANDWIDTH LIMITATION}

It was mentioned in Section II that cubic-term generator architectures made of multiple nonlinear operations represent a potential noise advantage over circuits which generate IM3 products using a single cubic nonlinearity due to the fact that the initial nonlinear products are only attenuated with respect to the noise floor as the square of the input signal rather than the cube. The reason for this is that the noise contributions of subsequent multiplier circuits can be made negligible by adding gain immediately after the initial squaring, as shown in Fig. 5.

Adding significant amounts of gain in between nonlinear operations can be problematic if all IM2 products after the initial squaring were required to be faithfully retained. For example, most of the relevant blockers in UMTS are clustered around $2 \mathrm{GHz}$ [9], and broadband circuitry operating up to 4 $\mathrm{GHz}$ is required to retain all IM2 products. Fortunately, this is not the case. Previous cubic-term generators [18], [19] proposed to cancel second-order harmonics while remaining effectively memoryless by using polyphase splitters and separate I and Q squaring circuits for the initial nonlinear operation. Although

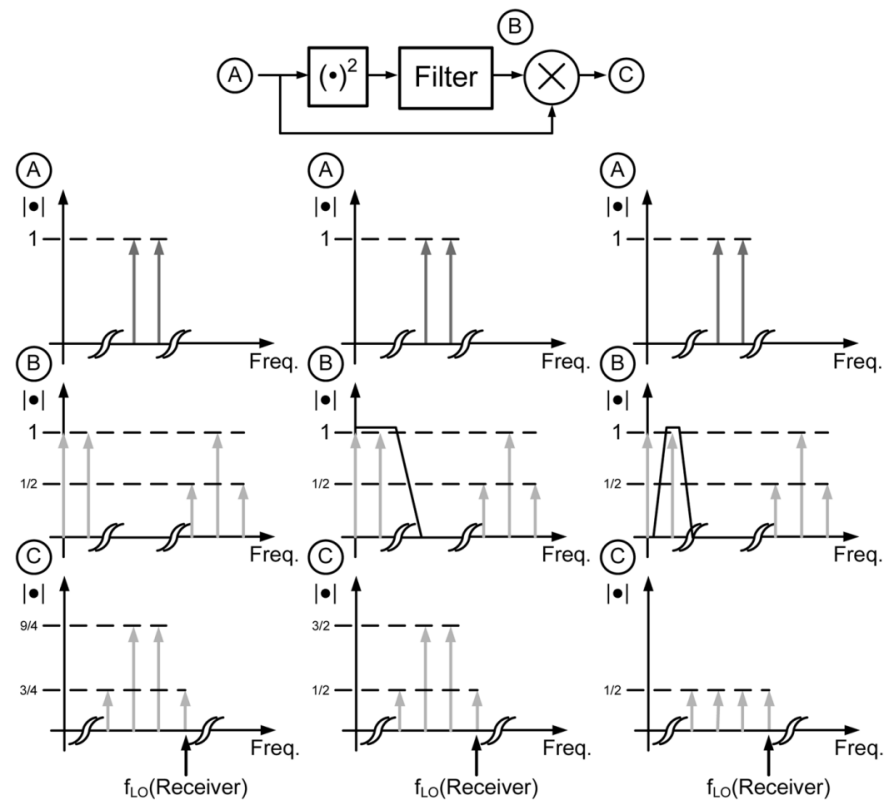

Fig. 6. Effects of baseband frequency response on IM3 products in a multistage cubic-term generator for a two-tone test.

an explicit filter can be used for this purpose, a design difficulty arises in that, if the cutoff frequency is made too low, the filter group delay results in an error term in the nonlinear operation [18]. On the other hand, if the cutoff frequency is made too high, the second-order harmonics experience uneven attenuation and are large enough to contribute to the final IM3 products, resulting in asymmetry between the upper and lower IM3 sidebands, as in Fig. 4. In the receiver of [9], however, these memory effects can, at least, in principle, be undone with a multitap adaptive equalizer. Hence, one or more bandlimited gain stages can be placed in between nonlinear operations without strict attention to the interstage frequency response characteristics.

It also turns out that, unlike in transmit predistortion circuits, retaining the IM2 products that fall around DC is not required once higher frequency IM2 products and harmonics are strongly attenuated. This is because, in the receiver, the precise relation of all of the third-order distortion components is unimportant; the only requirement is that the proper IM3 products occur around $f_{\mathrm{LO}}$. This difference is shown in Fig. 6 using a two-tone test to represent a more arbitrary modulated signal. In the second column, all of the third-order distortion products have the proper relative amplitude and can be used to cancel all of the terms arising from third-order nonlinearities in a predistortion scheme. In the third column, the inner and outer IM3 products no longer have the proper relationship to allow the concurrent cancellation of all terms. Although such a reference signal could function in order to cancel only the outer IM3 products in a predistorter two-tone proxy linearity test, for a practical modulated signal, all of the third-order products are important, and an improper relation between the terms would manifest itself in adjacent channel power ratio and error vector magnitude tests.

Therefore, only IM2 products around the beat frequency in a two-tone test need to be retained, as shown in Fig. 7. This is a significant advantage in the circuit in Fig. 3, as the peak IM2 products around DC can be larger than the peak IM2 products around the beat frequency, dictating the compression point 


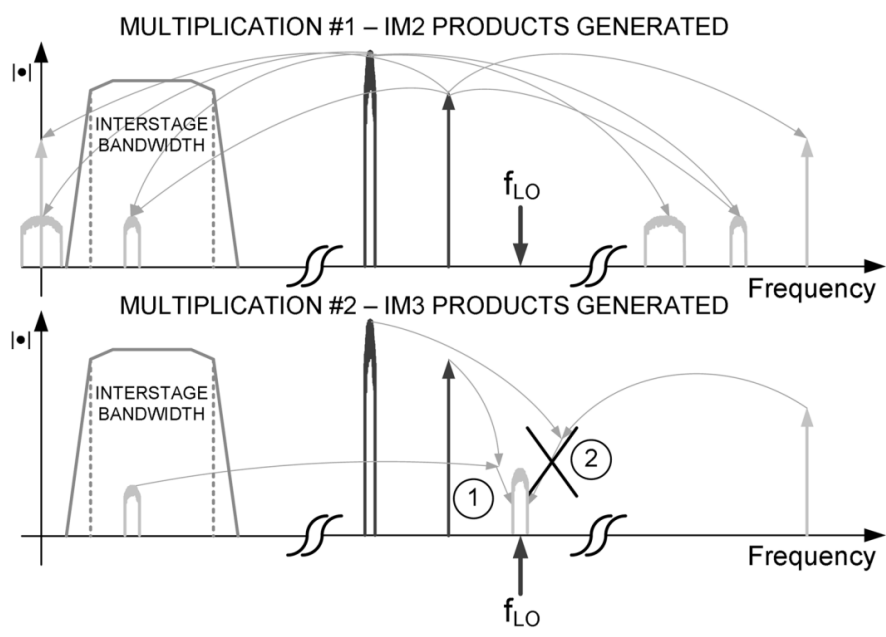

Fig. 7. Frequency domain depiction of proposed cubic-term generator internal bandwidth limitation.

of the cubic-term generator without contributing to the desired IM3 output products. For FDD UMTS Region 1, it can be calculated that the required interstage bandwidth, denoted $f_{\mathrm{IS}}$, is 185 $\mathrm{MHz}$ centered around 157.5 MHz [9]. As shown in Fig. 6, the relationship between the four output IM3 products in this case is not the same as that in the output of a true cubing circuit. As a result, the circuit of Fig. 3 must be described as a "cubic-term generator."

Although the linearity requirements of the FDD UMTS Region 1 specification [23] are dictated by nonlinear interactions between only two signals (transmitter (TX) leakage and a continuous-wave (CW) blocker), the cubic-term generator should also be able to handle the more general case of problematic IM3 products arising as a result of three arbitrary bandpass signals. A quick proof is presented in the following that shows that, in such a situation, a signal around only one beat frequency needs to be retained in order to reproduce the proper IM3 products. The proof sketch is as follows.

1) Consider three modulated signals, each at different frequencies, such that an IM3 product falls at $f_{\mathrm{LO}}=f_{A}+$ $f_{B}-f_{C}$.

2) A signal content at only three out of the ten possible IM2 frequencies might possibly need to be retained: $f_{A}+f_{B}$, $\left|f_{A}-f_{C}\right|$, and $\left|f_{B}-f_{C}\right|$.

3) The goal is to show that the IM3 products produced by multiplying each of these IM2 components by the original set of three modulated signals are the same. In this case, the signal content near only one of these three relevant IM2 frequencies needs to be retained to properly reproduce the IM3 products at $f_{\mathrm{LO}}$.

4) In order to do this, one of the two beat frequencies is chosen, and the IM2 products at that frequency are computed.

5) Next, the signal content at this frequency is multiplied by the signal not involved in the aforementioned IM2 products, and the relevant IM3 signal content at $f_{\mathrm{LO}}$ is retained.

6) Next, the IM2 products at $f_{A}+f_{B}$ are computed and multiplied by the signal at $f_{C}$. The resultant terms at $f_{\mathrm{LO}}$ should equal the terms computed in step 5) if these high-frequency terms can be discarded.
7) Finally, if only one of the two beat-frequency terms is necessary, then the letters A and B can be interchanged in the expression obtained in steps 5) and 6) with no change in the expression.

8) Note that the analysis shown below is indifferent as to whether difference terms such as $f_{A}-f_{C}$ are greater than or less than zero. Hence, this analysis is general for the various permutations of relative blocker frequency locations.

Elaboration on this sketch commences at step 4), and the IM2 terms at $f_{A}-f_{C}$ are computed. Recall that the bandpass signals may be expressed as

$$
\begin{aligned}
& s_{A}(t)=s_{\mathrm{AI}}(t) \cos \left(2 \pi f_{A} t\right)+s_{\mathrm{AQ}}(t) \sin \left(2 \pi f_{A} t\right) \\
& s_{C}(t)=s_{\mathrm{CI}}(t) \cos \left(2 \pi f_{C} t\right)+s_{\mathrm{CQ}}(t) \sin \left(2 \pi f_{C} t\right) .
\end{aligned}
$$

Multiplying these two signals together and applying the relevant trigonometric identities yields four separate terms

$$
\begin{aligned}
s_{A}(t) s_{C}(t)= & {\left[s_{\mathrm{AI}}(t) s_{\mathrm{CI}}(t)+s_{\mathrm{AQ}}(t) s_{\mathrm{CQ}}(t)\right] } \\
& \times \cos \left(2 \pi\left(f_{A}-f_{C}\right) t\right) \\
& +\left[s_{\mathrm{AQ}}(t) s_{\mathrm{CI}}(t)-s_{\mathrm{AI}}(t) s_{\mathrm{CQ}}(t)\right] \\
& \times \sin \left(2 \pi\left(f_{A}-f_{C}\right) t\right) \\
& +\left[s_{\mathrm{AI}}(t) s_{\mathrm{CI}}(t)-s_{\mathrm{AQ}}(t) s_{\mathrm{CQ}}(t)\right] \\
& \times \cos \left(2 \pi\left(f_{A}+f_{C}\right) t\right) \\
& +\left[s_{\mathrm{AI}}(t) s_{\mathrm{CQ}}(t)+s_{\mathrm{AQ}}(t) s_{\mathrm{CI}}(t)\right] \\
& \times \sin \left(2 \pi\left(f_{A}+f_{C}\right) t\right) .
\end{aligned}
$$

Taking the top two terms and multiplying by $s_{B}(t)$ yields a set of IM3 products at $f_{\mathrm{LO}}$

$$
\begin{aligned}
& {\left[s_{B}(t)\left[s_{A}(t) s_{C}(t)\right]_{f_{A}-f_{C}}\right]_{f_{A}+f_{B}-f_{C}}} \\
& =\left[s_{\mathrm{AI}}(t) s_{\mathrm{CI}}(t)+s_{\mathrm{AQ}}(t) s_{\mathrm{CQ}}(t)\right] \times s_{\mathrm{BI}}(t) \cos \left(2 \pi f_{\mathrm{LO}} t\right) \\
& \quad+\left[s_{\mathrm{AI}}(t) s_{\mathrm{CQ}}(t)-s_{\mathrm{AQ}}(t) s_{\mathrm{CI}}(t)\right] \times s_{\mathrm{BQ}}(t) \cos \left(2 \pi f_{\mathrm{LO}} t\right) \\
& \quad+\left[s_{\mathrm{AI}}(t) s_{\mathrm{CI}}(t)+s_{\mathrm{AQ}}(t) s_{\mathrm{CQ}}(t)\right] \times s_{\mathrm{BQ}}(t) \sin \left(2 \pi f_{\mathrm{LO}} t\right) \\
& \quad+\left[s_{\mathrm{AQ}}(t) s_{\mathrm{CI}}(t)-s_{\mathrm{AI}}(t) s_{\mathrm{CQ}}(t)\right] \times s_{\mathrm{BI}}(t) \sin \left(2 \pi f_{\mathrm{LO}} t\right) .
\end{aligned}
$$

Moving on to step 6), the last two terms of (6) are retained and $\mathrm{C}$ is replaced with $\mathrm{B}$ to obtain the relevant high-frequency IM2 products

$$
\begin{aligned}
{\left[s_{A}(t) s_{B}(t)\right]_{f_{A}+f_{B}}=} & {\left[s_{\mathrm{AI}}(t) s_{\mathrm{BI}}(t)-s_{\mathrm{AQ}}(t) s_{\mathrm{BQ}}(t)\right] } \\
& \times \cos \left(2 \pi\left(f_{A}+f_{B}\right) t\right) \\
& +\left[s_{\mathrm{AI}}(t) s_{\mathrm{BQ}}(t)+s_{\mathrm{AQ}}(t) s_{\mathrm{BI}}(t)\right] \\
& \times \sin \left(2 \pi\left(f_{A}+f_{B}\right) t\right) .
\end{aligned}
$$

Multiplying by $s_{C}(t)$ and taking the terms at $f_{\mathrm{LO}}$ yields another set of IM3 products at $f_{\mathrm{LO}}$

$$
\begin{aligned}
& {\left[s_{C}(t)\left[s_{A}(t) s_{B}(t)\right]_{f_{A}+f_{B}}\right]_{f_{A}+f_{B}-f_{C}}} \\
& =\left[s_{\mathrm{AI}}(t) s_{\mathrm{BI}}(t)-s_{\mathrm{AQ}}(t) s_{\mathrm{BQ}}(t)\right] s_{\mathrm{CI}}(t) \cos \left(2 \pi f_{\mathrm{LO}} t\right) \\
& \quad+\left[s_{\mathrm{AI}}(t) s_{\mathrm{BQ}}(t)+s_{\mathrm{AQ}}(t) s_{\mathrm{BI}}(t)\right] s_{\mathrm{CQ}}(t) \cos \left(2 \pi f_{\mathrm{LO}} t\right) \\
& \quad+\left[s_{\mathrm{AQ}}(t) s_{\mathrm{BQ}}(t)-s_{\mathrm{AI}}(t) s_{\mathrm{BI}}(t)\right] s_{\mathrm{CQ}}(t) \sin \left(2 \pi f_{\mathrm{LO}} t\right) \\
& \quad+\left[s_{\mathrm{AQ}}(t) s_{\mathrm{BI}}(t)+s_{\mathrm{AI}}(t) s_{\mathrm{BQ}}(t)\right] s_{\mathrm{CI}}(t) \sin \left(2 \pi f_{\mathrm{LO}} t\right) .
\end{aligned}
$$


Rearranging (7) and (9) shows that they are the same expression

$$
\begin{aligned}
{\left[s_{A}(t)\right.} & \left.s_{B}(t) s_{C}(t)\right]_{f_{A}+f_{B}-f_{C}} \\
= & {\left[s_{\mathrm{AI}}(t) s_{\mathrm{BI}}(t) s_{\mathrm{CI}}(t)-s_{\mathrm{AQ}}(t) s_{\mathrm{BQ}}(t) s_{\mathrm{CI}}(t)\right.} \\
& \left.+s_{\mathrm{AI}}(t) s_{\mathrm{BQ}}(t) s_{\mathrm{CQ}}(t)+s_{\mathrm{AQ}}(t) s_{\mathrm{BI}}(t) s_{\mathrm{CQ}}(t)\right] \\
& \times \cos \left(2 \pi f_{\mathrm{LO}} t\right) \\
& +\left[s_{\mathrm{AQ}}(t) s_{\mathrm{BQ}}(t) s_{\mathrm{CQ}}(t)-s_{\mathrm{AI}}(t) s_{\mathrm{BI}}(t) s_{\mathrm{CQ}}(t)\right. \\
& \left.+s_{\mathrm{AQ}}(t) s_{\mathrm{BI}}(t) s_{\mathrm{CI}}(t)+s_{\mathrm{AI}}(t) s_{\mathrm{BQ}}(t) s_{\mathrm{CI}}(t)\right] \\
& \times \sin \left(2 \pi f_{\mathrm{LO}} t\right) .
\end{aligned}
$$

Since the letters A and B can be interchanged in (10) with no resultant change to the expression, it does not matter which of the two beat-frequency terms is originally retained. This satisfies the requirements of step 7), and hence, the proof is complete.

\section{Noise in Multistage Cubic-Term Generators}

As mentioned in Section $\mathrm{V}$, the target value of $I E R_{\mathrm{CUB}}$ under peak blocking is $34 \mathrm{~dB}$ for a UMTS receiver with an uncorrected IIP3 of $-9 \mathrm{dBm}$. In a conservative design, the INR should dominate the IER, as accurately predicting higher order nonlinear terms requires precise device modeling at high frequencies, which cannot always be guaranteed. To determine the INR of the proposed cubic-term generator architecture shown in Fig. 3, the INR of the initial squaring circuit is analyzed for the effective two-tone test imposed by the UMTS Region 1 out-of-band blocking requirement and is referred to the input of the active balun. The key assumption here is that the noise of these input devices dominates that of the entire circuit under the conditions of interest, namely, under peak blocking.

Assuming that the transconductances of the MOS devices can be expressed as a Taylor series (11), the beat-frequency IM2 product voltage at the balun input can be expressed as (12) where $A_{X}$ represents the differential amplitude of sinusoidal blocker $\mathrm{X}$ and $R$ represents the load resistor of the circuit input stage. Here, the UMTS TX leakage is modeled as sinusoidal blocker \#2

$$
\begin{aligned}
I_{d}\left(v_{\mathrm{in}}\right) & =a_{0}+a_{1} v_{\mathrm{in}}+a_{2} v_{\mathrm{in}}^{2}+a_{3} v_{\mathrm{in}}^{3}+O(4) \\
I_{\mathrm{RMS}}^{2} & =\frac{1}{8}\left(a_{2} A_{1} A_{2} R\right)^{2} .
\end{aligned}
$$

The value of $a_{2}$ can be found via the Taylor series expansion of the MOS short-channel current (13) [23]. To simplify the notation, the equalities in (14) have been substituted

$$
\begin{aligned}
I_{d}(x) & =K B\left(V_{\mathrm{od}}+x\right)^{2}\left(V_{\mathrm{od}}+x+B\right)^{-1} \\
V_{\mathrm{od}} & =V_{\mathrm{GS}}-V_{\mathrm{TH}} \quad K=\frac{1}{2} \frac{W}{L} \mu_{n} C_{\mathrm{ox}} \quad B=L E_{\mathrm{sat} .}
\end{aligned}
$$

Taking the repeated derivatives of (13) yields the following:

$$
\begin{aligned}
& g_{m}=a_{1}=\left.\frac{\partial I_{d}}{\partial x}\right|_{x=0}=\frac{I_{d}}{V_{\mathrm{od}}} \cdot \frac{V_{\mathrm{od}}+2 B}{V_{\mathrm{od}}+B} \\
& a_{2}=\left.\frac{1}{2 !} \frac{\partial^{2} I_{d}}{\partial x^{2}}\right|_{x=0}=\frac{K B}{\left(V_{\mathrm{od}}+B\right)}\left(1-\frac{V_{\mathrm{od}}}{V_{\mathrm{od}}+B}\right)^{2}
\end{aligned}
$$

The power spectral density (PSD) of the thermal noise of the input devices is evaluated using the MOS long-channel noise model. This was done as the simulation models available for this work permitted a more realistic and constant value of the noise $\gamma$ parameter over $V_{\text {od }}$ than did the short-channel noise model. In this case, the PSD at the input of the balun is given by (17), where $g_{m}$ is the transconductance of one of the input devices and $k$ is the Boltzmann's constant. Note that the dummy input devices contribute to this quantity as well

$$
N_{\mathrm{RMS}}^{2}(\Delta f)=4 k T R\left(4 \gamma g_{m} R+2\right) .
$$

As shown in Fig. 8, the thermal noise of the input devices referred to the input of the Gilbert multiplier has a bandpass characteristic due to the frequency response discussed in the previous section. Clearly, the noise around the desired beat-frequency IM2 products is upconverted to the IM3 products at RF by one of the blockers. Here, it is assumed that this is done by blocker 1 . However, any blocker signals falling within $\left\{f_{\mathrm{LO}}-\right.$ $\left.f_{\mathrm{IS}}, f_{\mathrm{LO}}+f_{\mathrm{IS}}\right\}$ will also contribute bandpass noise to the final INR quantity, even if they do not participate in the generation of IM3 products. To refer this noise back to the input of the balun stage, it suffices to realize that, assuming local bandpass gain $G_{X}$, the noise PSD upconverted and contributed by blocker X is given by $G_{X}^{2} A_{X}^{2} N_{\mathrm{RMS}}^{2}(\Delta f)$. Integrating the double-sided desired signal bandwidth $f_{\mathrm{BW}}=3.84 \mathrm{MHz}$ yields the total noise power. To refer back to the input of the balun, each noise contribution must be divided by $G_{1}^{2} A_{1}^{2}$. In this case, the INR is given by (18), where $M$ represents the total number of blockers in the relevant frequency range

$$
\begin{aligned}
& I N R=\frac{I_{d} B^{4} A_{1}^{4} A_{2}^{2}}{64 k T f_{\mathrm{BW}}\left(\sum_{i=1}^{M}\left(\frac{G_{i}}{G_{1}} A_{i}\right)^{2}\right) V_{\mathrm{od}}^{3}\left(V_{\mathrm{od}}+B\right)^{3}} \\
& \times \frac{1}{2 \gamma\left(V_{\mathrm{od}}+2 B\right)+\frac{1}{R} \frac{V_{\mathrm{od}}\left(V_{\mathrm{od}}+B\right)}{I_{d}}} .
\end{aligned}
$$

Although the summation in (18) seems to suggest that INR can be arbitrarily low depending on how many blockers exist, in practice, the total blocker power is bounded and, for a real-world 


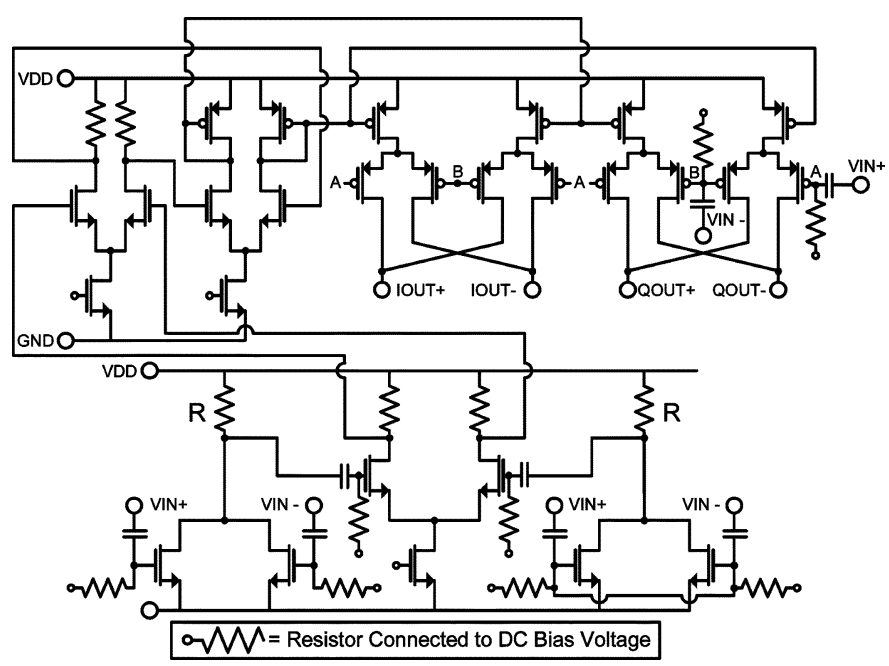

Fig. 9. Schematic of cubic-term generator used for simulation.

deployment, would have to be estimated by field measurements in the relevant frequency range. For the UMTS specification tests of interest in [9], however, only the TX leakage and a CW blocker are present at any given time and $M=2$. Similarly, it would seem that the optimal design strategy would be to set $V_{\text {od }}$ as low as possible by grounding the input device gates and increasing device width until enough signal is obtained. However, it can be shown [25] that, as $V_{\text {od }}$ approaches $0 \mathrm{~V}$ well into the weak inversion regime, the ratio of IM2/IM4 reaches a maximum, after which it rapidly falls off. This quantity represents a practical lower bound on $V_{\text {od }}$.

The only remaining task is to find the relationship between the current of each input device and the remainder of the circuit. A simple way to do this is to render the current draw of each gain, balun, and Gilbert cell stage equal. To suppress the noise of the Gilbert cell adequately in this case requires multiple gain stages, as shown in the circuit in Fig. 9, which is used to compare simulation versus calculation. For simulations, $I_{D}$, ТОТ $=24 I_{D}$ was chosen to suppress the noise of the balun and Gilbert cell devices adequately.

To confirm the calculations, they are compared with simulations in Fig. 10 for $L=0.6 \mu \mathrm{m}, I_{D, \text { ТОT }}=1 \mathrm{~mA}, T=298 \mathrm{~K}$, and swept $V_{\text {od }}$ in a deep submicrometer process. A relatively large value of $L$ is targeted in order to improve matching and to minimize linear term feedthrough. Values for $\gamma$ and $E_{\text {sat }}$ were extracted from simulated device models and found to be 0.55 and $1.8 \mathrm{~V} / \mu \mathrm{m}$, respectively. The value of $\gamma$ is consistent with other recent reports of submicrometer CMOS devices [26], [27] but a bit lower, likely due to device operation near weak inversion and with relatively low $V_{\mathrm{ds}} . G_{2} / G_{1}$ was simulated and found to be 0.8 . Fig. 11 shows the results of simulation versus calculation for $V_{\text {od }}=132 \mathrm{mV}, I_{D \text {, ТОT }}=1 \mathrm{~mA}, T=298 \mathrm{~K}$, and swept $A_{2} / A_{1}$. In both Figs. 10(b) and 11(b), the simulated INR of both the input stage and total circuit is shown. The data points at $A_{2}=-26 \mathrm{dBV}$ (TX leakage model) in Fig. 10 represent peak blocking for the receiver of [9], and it can be seen here that the input stage indeed dominates the INR performance of the circuit. Furthermore, for most presented values of $V_{\text {od }}$, the circuit meets the target INR value of $34 \mathrm{~dB}$ for $1 \mathrm{~mA}$ of current. As this current draw is much less than the $28 \mathrm{~mA}$ consumed by the original receiver path presented in [9], it represents a good
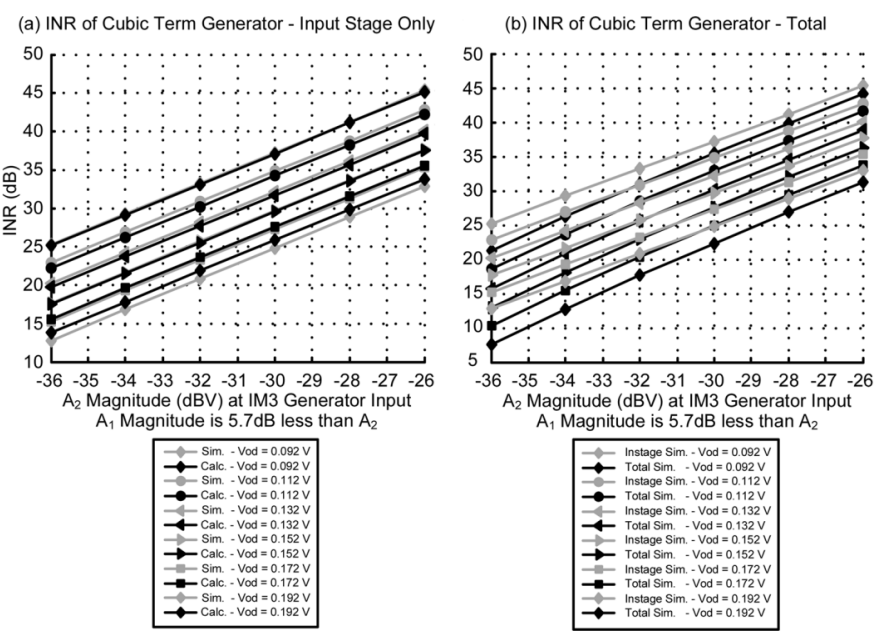

Fig. 10. (a) Calculated versus simulated INR due to input stage components swept over $V_{\text {od }}$. (b) Simulated INR, input stage, and total swept over $V_{\text {od }}$.
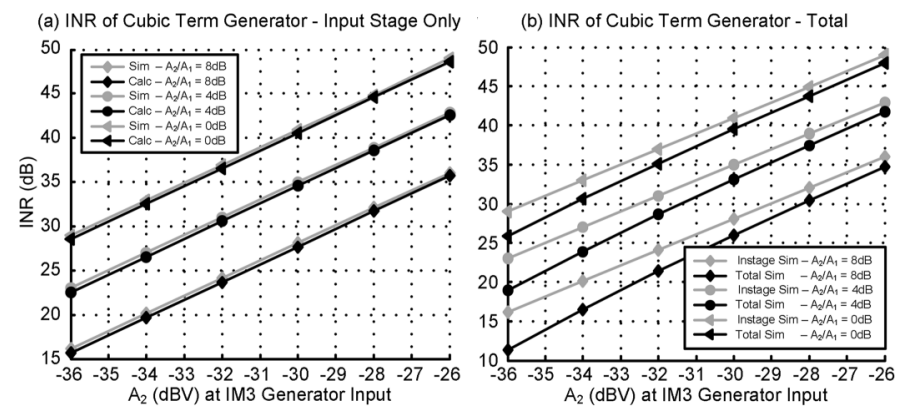

Fig. 11. (a) Calculated versus simulated INR due to input stage components swept over $A 2 / A 1$ ratio. (b) Simulated INR, input stage, and total swept over $A 2 / A 1$ ratio.

design option for a portion of the alternate path enhancement circuitry.

\section{ERRor DUE TO INTERSTAGE DELAY}

Although the effective elimination of many IM2 products due to bandwidth limitations does not contribute to error in the desired IM3 products at the output of the cubic-term generator, the group delay resulting from the nonconstant frequency response experienced by the beat-frequency products does. Specifically, the group delay between multiplications in the IM3 term generator may result in a modulated signal multiplying a delayed version of itself or another modulated signal. This can be seen by considering an unfiltered BPSK-modulated signal, as shown in Fig. 12. In this case, the IM3 products consist of a frequencytranslated version of the squared BPSK signal. Error due to the delay occurs in the time interval over which adjacent symbols both differ and overlap, denoted by the dashed lines. The instance of this effect which occurs under the FDD UMTS Region 1 specification [23] is an IM3 product arising from the squared QPSK TX leakage and a CW tone ranging from 1.67 to $1.85 \mathrm{GHz}$.

The PSD of a squared complex digitally modulated signal has been studied in the past [28]; however, it is vital to perform a similar analysis for QPSK here for two reasons. First, unlike the squared offset QPSK signal evaluated in [28], the PSDs of the squared QPSK modulated signal I and Q components at the desired signal frequency are different and, on average, retain 


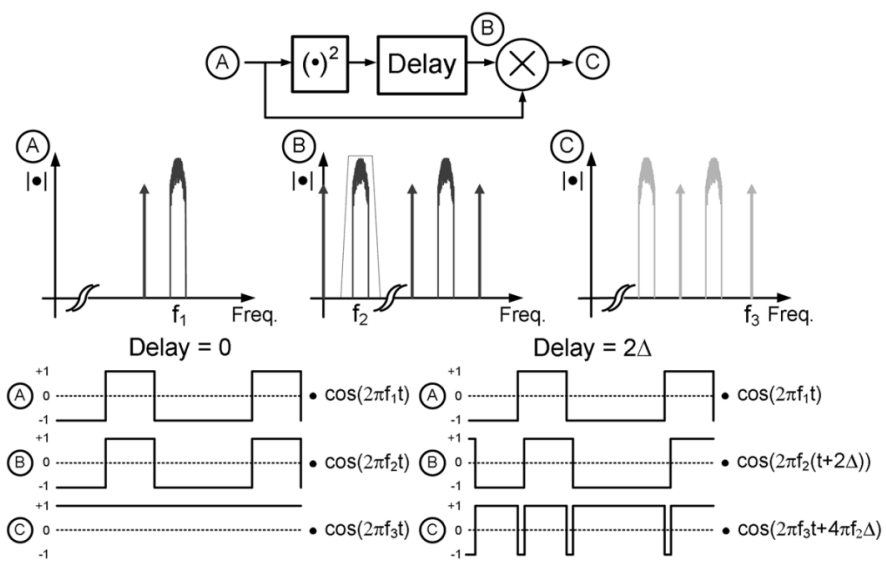

Fig. 12. Depiction of the effect of interstage group delay on CW and BPSK blocking signals producing IM3 products.

more power within the original signal bandwidth. Second, the analysis needs to be performed taking into account the delay between multiplications in a multistage cubic-term generator in order to compute the error due to this effect. The authors of [18] correctly noted that, in a predistortion system with only gain and phase adaptive adjustments, the achievable cancellation of IM3 products is given by a bandpass first-order noise-shaping function. In the receiver, however, half the value of this delay is assumed to be reversed by the multitap adaptive equalizer along with any gain and phase mismatch in the process of minimizing mean square error. In this case, the integrated in-band error will be lower than that given by [18].

The statistical analysis begins by recognizing that a bandpass complex modulated signal can be represented as follows, where $A_{m}$ is the modulated signal carrier amplitude and $f_{C}$ is the modulated signal carrier frequency:

$$
x(t)=A_{m} v(t) \cos \left(2 \pi f_{C} t\right)+A_{m} w(t) \sin \left(2 \pi f_{C} t\right)
$$

As in [28], the TX channel filter is approximated with a brick wall frequency response with a cutoff frequency of $1 / 2 T$, where $T$ equals the chip rate of the interfering modulation. Hence, for QPSK modulation, the signals are expressed as follows, where $\alpha$ is a random time shift uniformly distributed in $(0, T)$ :

$$
\begin{aligned}
v(t) & =\sum_{n=-\infty}^{\infty} i_{n} \operatorname{sinc}\left(\frac{t-n T+\alpha}{T}\right) \\
w(t) & =\sum_{n=-\infty}^{\infty} q_{n} \operatorname{sinc}\left(\frac{t-n T+\alpha}{T}\right)
\end{aligned}
$$

For the sake of this analysis, $i_{n}$ and $q_{n}$ are independent identically distributed discrete random variables realizing one of $\{1,-1\}$. To begin the computation of the IM3 component of interest, the bandpass modulated signal must be squared and its high-pass components retained, yielding the following:

$$
\begin{array}{r}
y(t)=A_{m}^{2}\left[\frac{1}{2} v^{2}(t) \cos \left(4 \pi f_{C} t\right)\right. \\
+v(t) w(t) \sin \left(4 \pi f_{C} t\right) \\
\left.-\frac{1}{2} w^{2}(t) \cos \left(4 \pi f_{C} t\right)\right]
\end{array}
$$

To generate the relevant IM3 products at baseband, the expression in (21) is multiplied by two CW signals, a blocker, and an LO signal. This constitutes an effective multiplication by a single sinusoidal signal with an arbitrary phase $\Theta$ with respect to the carrier terms of the modulated signal $s(t)=$ $A_{\mathrm{CW}} \cos \left(4 \pi f_{C} t+\Theta\right)$ and yields after low-pass filtering at baseband

$$
\begin{aligned}
z(t)=\frac{1}{4} A_{m}^{2} A_{\mathrm{CW}}[ & {\left[v^{2}(t)-w^{2}(t)\right] \cos (\Theta) } \\
& -2 v(t) w(t) \sin (\Theta)] .
\end{aligned}
$$

Note that this signal appears at the I channel baseband. In order to find the signal that appears at the $\mathrm{Q}$ channel baseband, it is merely sufficient to substitute $\Theta=\Theta+90^{\circ}$ into the final expression. The order of multiplications aforementioned is taken to simplify the analysis and is not the same as that performed in the circuit in Fig. 3. However, the proof in Section $\mathrm{V}$ can be used to show that the result is the same in either case.

In the alternate path, (22) is altered by the presence of the interstage group delay, which is denoted by $2 \Delta$ to yield the following:

$$
\begin{aligned}
z^{\prime}(t)= & \frac{1}{4} A_{m}^{2} A_{\mathrm{CW}} \\
& \times[[v(t+\Delta) v(t-\Delta)-w(t+\Delta) w(t-\Delta)] \cos (\Theta) \\
& -[v(t+\Delta) w(t-\Delta)+w(t+\Delta) v(t-\Delta)] \sin (\Theta)] .
\end{aligned}
$$

Expressing (23) in this fashion makes an implicit assumption that the group delay is constant across the entire band of frequencies spanned by the IM2 beat-frequency products. This assumption is reasonable, at least for the conditions directly of interest - the simulation results of the cubic-term generator in [9] show that the average group delay deviation within the interstage frequency band over the 3.84-MHz TX leakage bandwidth is $0.67 \%$. In this case, the error due to the delay is given by (24). Starting from (24), the time parameter is removed from the arguments of $v$ and $w$, while $\Delta$ is relegated to a subscript to condense the expressions

$$
\begin{aligned}
z(t)-z^{\prime}(t)= & e(t) \\
= & \frac{1}{4} A_{m}^{2} A_{\mathrm{CW}}\left[\left[\left(v^{2}-v_{\Delta} v_{-\Delta}\right)\right.\right. \\
& \left.-\left(w^{2}-w_{\Delta} w_{-\Delta}\right)\right] \cos (\Theta) \\
& \left.-\left[2 v w-v_{\Delta} w_{-\Delta}-w_{\Delta} v_{-\Delta}\right] \sin (\Theta)\right] .
\end{aligned}
$$

The autocorrelation of the error is therefore given by the following, where the delay offset $\tau$ is also placed within the subscripts of $v$ and $w$ where it exists:

$$
\begin{aligned}
& R_{e e}(t+\tau ; t) \\
& =\frac{1}{16} A_{m}^{4} A_{\mathrm{CW}}^{2} \\
& \quad \times E\left[\left[\left[\left(v_{0}^{2}-v_{\tau+\Delta} v_{\tau-\Delta}\right)-\left(w_{\tau}^{2}-w_{\tau+\Delta} w_{\tau-\Delta}\right)\right]\right.\right. \\
& \left.\quad \times \cos (\Theta)-\left[2 v_{\tau} w_{\tau}-v_{\tau+\Delta} w_{\tau-\Delta}-w_{\tau+\Delta} v_{\tau-\Delta}\right] \sin (\Theta)\right] \\
& \quad \times\left[\left[\left(v^{2}-v_{\Delta} v_{-\Delta}\right)-\left(w^{2}-w_{\Delta} w_{-\Delta}\right)\right] \cos (\Theta)\right. \\
& \left.\left.\quad-\left[2 v w-v_{\Delta} w_{-\Delta}-w_{\Delta} v_{-\Delta}\right] \sin (\Theta)\right]\right] .
\end{aligned}
$$

Note that terms such as $E[\bullet](\sin \Theta)(\cos \Theta)$ are equal to zero due to the fact that one out of the four terms is a zero-mean bit 
sequence that is independent of the other three bit sequences. Multiplying out only the terms resulting in $E[\bullet]\left(\cos ^{2} \Theta\right)$ and $E[\bullet]\left(\sin ^{2} \Theta\right)$ yields the following:

$$
\begin{aligned}
E\left[v_{\tau}^{2} v^{2}-v_{\tau}^{2} v_{\Delta} v_{-\Delta}\right. & \\
& -v_{\tau}^{2} w^{2}+v_{\tau}^{2} w_{\Delta} w_{-\Delta}-v_{\tau+\Delta} v_{\tau-\Delta} v^{2} \\
& +v_{\tau+\Delta} v_{\tau-\Delta} v_{\Delta} v_{-\Delta}+v_{\tau+\Delta} v_{\tau-\Delta} w^{2} \\
& -v_{\tau+\Delta} v_{\tau-\Delta} w_{\Delta} w_{-\Delta}-w_{\tau}^{2} v^{2} \\
& +w_{\tau}^{2} v_{\Delta} v_{-\Delta}+w_{\tau}^{2} w^{2} \\
& -w_{\tau}^{2} w_{\Delta} w_{-\Delta}+w_{\tau+\Delta} w_{\tau-\Delta} v^{2} \\
& -w_{\tau+\Delta} w_{\tau-\Delta} v_{\Delta} v_{-\Delta}-w_{\tau+\Delta} w_{\tau-\Delta} w^{2} \\
& \left.+w_{\tau+\Delta} w_{\tau-\Delta} w_{\Delta} w_{-\Delta}\right] \cdot \cos ^{2}(\Theta) \\
= & C_{0}-A-D_{0}+B-A_{\mathrm{FR}}+C \\
& +B_{\mathrm{FR}}-D-D_{0}+B+C_{0}-A \\
& \left.+B_{\mathrm{FR}}-D-A_{\mathrm{FR}}+C\right]\left(\frac{1}{2}+\frac{1}{2} \cos (2 \Theta)\right) \\
E\left[4 v_{\tau}\right. & w_{\tau} v w_{-}-2 v_{\tau} w_{\tau} v_{\Delta} w_{-\Delta}-2 v_{\tau} w_{\tau} w_{\Delta} v_{-\Delta} \\
& -2 v_{\tau+\Delta} w_{\tau-\Delta} v w_{+} v_{\tau+\Delta} w_{\tau-\Delta} v_{\Delta} w_{-\Delta} \\
& +v_{\tau+\Delta} w_{\tau-\Delta} w_{\Delta} v_{-\Delta}-2 w_{\tau+\Delta} v_{\tau-\Delta} v w \\
& \left.+w_{\tau+\Delta} v_{\tau-\Delta} v_{\Delta} w_{-\Delta}+v_{\tau+\Delta} w_{\tau-\Delta} w_{\Delta} v_{-\Delta}\right] \sin ^{2}(\Theta) \\
=[ & 4 F_{0}-2 E-2 E-2 E_{\mathrm{FR}}+F \\
& \left.+G-2 E_{\mathrm{FR}}+G+F\right]\left(\frac{1}{2}-\frac{1}{2} \cos (2 \Theta)\right)
\end{aligned}
$$

A brief discussion on the notation in (26) and (27) is in order. Terms with the same letter can be shown to have the same PSD. The terms subscripted with zero denote the terms that constitute the original IM3 signal. The PSD of the original IM3 products can therefore be obtained by setting $\Delta=0$ in the PSD of C, D, and F. The terms denoted with FR can be shown to have PSDs equivalent to their respective terms, with the exception that the PSD is frequency reversed.

The computation continues by evaluating each of these terms in turn to obtain subexpressions that can be made time independent such that their Fourier transforms can be taken. Term $\mathrm{C}$ is evaluated first in the following:

$$
\begin{aligned}
& C(t+\tau ; t) \\
& =\sum_{n, m, p, r=-\infty}^{\infty} E\left[i_{n} i_{m} i_{p} i_{r}\right] \frac{1}{T} \int_{0}^{T} \operatorname{sinc}\left(\frac{t+\tau-n T+\Delta+\alpha}{T}\right) \\
& \quad \times \operatorname{sinc}\left(\frac{t+\tau-m T-\Delta+\alpha}{T}\right) \operatorname{sinc}\left(\frac{t-p T+\Delta+\alpha}{T}\right) \\
& \quad \times \operatorname{sinc}\left(\frac{t-r T-\Delta+\alpha}{T}\right) \mathrm{d} \alpha .
\end{aligned}
$$

The term in the expected value operator evaluates to unity rather than zero under only the following conditions.

1) $n=m=p=r$.

2) $n=m=k$ and $p=r=l \neq n$.

3) $n=p=k$ and $m=r=l \neq n$.

4) $n=r=k$ and $m=p=l \neq n$.
These four cases yield the terms in (29), in respective order. In order to condense the expressions, "sinc" is replaced with " $s$ "

$$
\begin{aligned}
& C(t+\tau ; t) \\
& =-\frac{2}{T} \sum_{n=-\infty}^{\infty} \int_{0}^{T} s\left(\frac{t+\tau-n T+\Delta+\alpha}{T}\right) \\
& \times s\left(\frac{t+\tau-n T-\Delta+\alpha}{T}\right) \\
& \times s\left(\frac{t-n T+\Delta+\alpha}{T}\right) s\left(\frac{t-n T-\Delta+\alpha}{T}\right) \mathrm{d} \alpha \\
& +\frac{1}{T} \sum_{k, l=-\infty}^{\infty} \int_{0}^{T} s\left(\frac{t+\tau-k T+\Delta+\alpha}{T}\right) \\
& \times s\left(\frac{t+\tau-k T-\Delta+\alpha}{T}\right) \\
& \times s\left(\frac{t-l T+\Delta+\alpha}{T}\right) s\left(\frac{t-l T-\Delta+\alpha}{T}\right) \mathrm{d} \alpha \\
& +\frac{1}{T} \sum_{k, l=-\infty}^{\infty} \int_{0}^{T} s\left(\frac{t+\tau-k T+\Delta+\alpha}{T}\right) \\
& \times s\left(\frac{t-k T+\Delta+\alpha}{T}\right) \\
& \times s\left(\frac{t+\tau-l T-\Delta+\alpha}{T}\right) s\left(\frac{t-l T-\Delta+\alpha}{T}\right) \mathrm{d} \alpha \\
& +\frac{1}{T} \sum_{k, l=-\infty}^{\infty} \int_{0}^{T} s\left(\frac{t+\tau-k T+\Delta+\alpha}{T}\right) \\
& \times s\left(\frac{t-k T-\Delta+\alpha}{T}\right) \\
& \times s\left(\frac{t+\tau-l T-\Delta+\alpha}{T}\right) s\left(\frac{t-l T+\Delta+\alpha}{T}\right) \mathrm{d} \alpha \\
& =\varepsilon+\gamma+\eta+\kappa \text {. }
\end{aligned}
$$

Note that the first term has a negative sign to account for the fact that three such terms need to be removed to avoid repeat counting of the same expression. The term A can be evaluated in a similar manner, resulting in the following:

$$
\begin{aligned}
A(t+ & \tau ; t) \\
= & \sum_{n, m, p, r=-\infty}^{\infty} E\left[i_{n} i_{m} i_{p} i_{r}\right] \\
& \times \frac{1}{T} \int_{0}^{T} s\left(\frac{t+\tau-n T+\alpha}{T}\right) s\left(\frac{t+\tau-m T+\alpha}{T}\right) \\
& \times s\left(\frac{t-p T+\Delta+\alpha}{T}\right) s\left(\frac{t-r T-\Delta+\alpha}{T}\right) \mathrm{d} \alpha \\
= & -\frac{2}{T} \sum_{n=-\infty}^{\infty} \int_{0}^{T} s\left(\frac{t+\tau-n T+\alpha}{T}\right) s\left(\frac{t+\tau-n T+\alpha}{T}\right) \\
& \times s\left(\frac{t-n T+\Delta+\alpha}{T}\right) s\left(\frac{t-n T-\Delta+\alpha}{T}\right) \mathrm{d} \alpha
\end{aligned}
$$




$$
\begin{aligned}
& +\frac{1}{T} \sum_{k, l=-\infty}^{\infty} \int_{0}^{T} s\left(\frac{t+\tau-k T+\alpha}{T}\right) s\left(\frac{t+\tau-k T+\alpha}{T}\right) \\
& \times s\left(\frac{t-l T+\Delta+\alpha}{T}\right) s\left(\frac{t-l T-\Delta+\alpha}{T}\right) \mathrm{d} \alpha \\
& +\frac{1}{T} \sum_{k, l=-\infty}^{\infty} \int_{0}^{T} s\left(\frac{t+\tau-k T+\alpha}{T}\right) s\left(\frac{t-k T+\Delta+\alpha}{T}\right) \\
& \times s\left(\frac{t+\tau-l T+\alpha}{T}\right) s\left(\frac{t-l T-\Delta+\alpha}{T}\right) \mathrm{d} \alpha \\
& +\frac{1}{T} \sum_{k, l=-\infty}^{\infty} \int_{0}^{T} s\left(\frac{t+\tau-k T+\alpha}{T}\right) \\
& \times s\left(\frac{t-k T-\Delta+\alpha}{T}\right) \\
& \times s\left(\frac{t+\tau-l T+\alpha}{T}\right) s\left(\frac{t-l T+\Delta+\alpha}{T}\right) \mathrm{d} \alpha \\
& =\lambda+\beta+2 \chi
\end{aligned}
$$

It can similarly be shown that

$$
\begin{array}{ll}
B=\beta & D=\gamma \\
E=\chi & F=\eta \\
G=\kappa . &
\end{array}
$$

The complete expression for the autocorrelation of the error signal can therefore be given by

$$
\begin{aligned}
R_{e e}(\tau)= & \frac{1}{16} A_{m}^{4} A_{\mathrm{CW}}^{2} \\
& \times\left(2\left[\varepsilon_{0}-\left(\lambda+\lambda_{\mathrm{TR}}\right)+\varepsilon\right]\left[\frac{1}{2}+\frac{1}{2} \cos (2 \Theta)\right]\right. \\
& \left.+2\left[2 \eta_{0}-2\left(\chi+\chi_{\mathrm{TR}}\right)+\eta+\kappa\right]\right)
\end{aligned}
$$

The complete expression for the autocorrelation of the original squared modulated signal can be given by

$$
R_{z z}(\tau)=\frac{1}{16} A_{m}^{4} A_{\mathrm{CW}}^{2}\left[\varepsilon_{0}[1+\cos (2 \Theta)]+4 \eta_{0}\right] .
$$

The general procedure for taking the Fourier transform of each of the five subterms can be shown in detail for term $\eta$. As many of the steps are repeated for each of the terms, they will only be performed for term $\eta$, while the unique steps for each term will be performed in detail.

1) Evaluation of Term $\eta$ : The first manipulation is to make the substitution that $l=k+s$, where $k$ and $s$ are both integers

$$
\begin{aligned}
\eta= & \frac{1}{T} \sum_{k, s=-\infty}^{\infty} \int_{0}^{T} s\left(\frac{t+\tau-k T+\Delta+\alpha}{T}\right) \\
& \times s\left(\frac{t+\tau-k T-s T-\Delta+\alpha}{T}\right) \\
& \times s\left(\frac{t-k T+\Delta+\alpha}{T}\right) \\
& \times s\left(\frac{t-k T-s T-\Delta+\alpha}{T}\right) \mathrm{d} \alpha .
\end{aligned}
$$

Next, the substitutions $t-k T+\alpha=u$ and $\mathrm{d} u=\mathrm{d} \alpha$ are made, and the appropriate adjustments to the limits of the integral are made

$$
\begin{aligned}
\eta=\frac{1}{T} \sum_{s=-\infty}^{\infty} \int_{-\infty}^{\infty} s & \left(\frac{u+\tau+\Delta}{T}\right) s\left(\frac{u+\tau-s T-\Delta}{T}\right) \\
& \times s\left(\frac{u+\Delta}{T}\right) s\left(\frac{u-s T-\Delta}{T}\right) \mathrm{d} u .
\end{aligned}
$$

Taking the Fourier transform of this expression yields

$$
\begin{aligned}
H(f)= & \frac{1}{T} \int_{-\infty}^{\infty} \sum_{s=-\infty}^{\infty} \int_{-\infty}^{\infty} s\left(\frac{u+\tau+\Delta}{T}\right) \\
& \times s\left(\frac{u+\tau-s T-\Delta}{T}\right) \\
& \times s\left(\frac{u+\Delta}{T}\right) s\left(\frac{u-s T-\Delta}{T}\right) \cdot \mathrm{d} u \mathrm{e}^{-j 2 \pi f \tau} \mathrm{d} \tau .
\end{aligned}
$$

Because it is known a priori that the PSD of the complete expression is a finite quantity at each frequency, integration and summation can be interchanged at will. Doing so yields the following steps:

$$
\begin{aligned}
H(f)= & \frac{1}{T} \sum_{s=-\infty}^{\infty} \int_{-\infty}^{\infty} s\left(\frac{u+\tau+\Delta}{T}\right) \\
& \times s\left(\frac{u+\tau-s T-\Delta}{T}\right) e^{-j 2 \pi f \tau} \mathrm{d} \tau \\
& \times \int_{-\infty}^{\infty} s\left(\frac{u+\Delta}{T}\right) s\left(\frac{u-s T-\Delta}{T}\right) \mathrm{d} u .
\end{aligned}
$$

Considering the two inner integrals separately, if the variable change $x=u+\tau$ is made, the following can be obtained:

$$
\begin{aligned}
H(f)= & \frac{1}{T} \sum_{s=-\infty}^{\infty} \int_{-\infty}^{\infty} s\left(\frac{x+\Delta}{T}\right) \\
& \times s\left(\frac{x-s T-\Delta}{T}\right) \mathrm{e}^{-j 2 \pi f x} \mathrm{~d} x \\
& \times \int_{-\infty}^{\infty} s\left(\frac{u+\Delta}{T}\right) \cdot s\left(\frac{u-s T-\Delta}{T}\right) e^{-j 2 \pi f u} \mathrm{~d} u
\end{aligned}
$$

Note that the Fourier transform of this sinc function is a rectangle function of total width $1 / T$ in frequency and of height $T$, denoted as $\Pi_{T}(f)$. Therefore, the Fourier transform of a sinc multiplied by a delayed sinc is a rectangle function convolved by a rectangle function which has been multiplied by a complex exponential. Performing this operation and successively interchanging integration and summation yields

$$
\begin{aligned}
H(f)= & \frac{1}{T} \int_{-\infty}^{\infty} \int_{-\infty}^{\infty} \sum_{s=-\infty}^{\infty} \mathrm{e}^{j 2 \pi(\varphi-\phi) s T} \\
& \times \Pi_{T}(f-\phi) \Pi_{T}(\phi) \mathrm{e}^{-j 4 \pi \phi \Delta} \mathrm{d} \phi \\
& \times \Pi_{T}(f-\varphi) \Pi_{T}(\varphi) \mathrm{e}^{j 4 \pi \varphi \Delta} \mathrm{d} \varphi .
\end{aligned}
$$


Recalling that the Fourier series of an impulse train is a sum of periodically spaced complex exponentials [29], the following change can be made to (39):

$$
\begin{aligned}
H(f)= & \frac{1}{T^{2}} \int_{-\infty}^{\infty} \int_{-\infty}^{\infty} \sum_{s=-\infty}^{\infty} \delta\left(\varphi-\phi-\frac{s}{T}\right) \\
& \times \Pi_{T}(f-\phi) \Pi_{T}(\phi) \\
& \times \Pi_{T}(f-\varphi) \Pi_{T}(\varphi) \mathrm{e}^{j 4 \pi(\varphi-\phi) \Delta} \mathrm{d} \varphi \mathrm{d} \phi
\end{aligned}
$$

Integration and summation can again be exchanged, and the inner integral can be evaluated using the sifting property of the Dirac delta function

$$
\begin{aligned}
H(f)= & \frac{1}{T^{2}} \int_{-\infty}^{\infty} \sum_{s=-\infty}^{\infty} \Pi_{T}(f-\phi) \Pi_{T}(\phi) \\
& \times \Pi_{T}\left(f-\phi-\frac{s}{T}\right) \Pi_{T}\left(\phi+\frac{s}{T}\right) \mathrm{e}^{j 4 \pi s \Delta / T} \mathrm{~d} \phi .
\end{aligned}
$$

Due to the strict bandlimited nature of the rectangle function, (41) only evaluates to a nonzero value for $s=0$. The summation and complex exponential thus disappear in the following:

$$
\begin{aligned}
H(f) & =\frac{1}{T^{2}} \int_{-\infty}^{\infty} \Pi_{T}^{2}(f-\phi) \Pi_{T}^{2}(\phi) \mathrm{d} \phi \\
& =\int_{-\infty}^{\infty} \Pi_{T}(f-\phi) \Pi_{T}(\phi) \mathrm{d} \phi=T \cdot \operatorname{Tr}(f) .
\end{aligned}
$$

It can be seen that $H(f)=H_{0}(f)$ and that the triangle function $\operatorname{Tr}(f)$ represents

$$
\operatorname{Tr}(f)= \begin{cases}|1-f T|, & 0 \leq|f| \leq \frac{1}{T} \\ 0, & \text { otherwise. }\end{cases}
$$

2) Evaluating Term $\kappa$ : Taking the Fourier transform of $\kappa$ and applying the steps outlined earlier results in

$$
\begin{aligned}
K(f)= & \frac{1}{T} \sum_{s=-\infty}^{\infty} \int_{-\infty}^{\infty} \Pi_{T}(f-\phi) \mathrm{e}^{j 2 \pi(f-\phi) \Delta} \\
& \times \Pi_{T}(\phi) \mathrm{e}^{-j 2 \pi \phi s T} \mathrm{e}^{-j 2 \pi \phi \Delta} \mathrm{d} \phi \\
& \times \int_{-\infty}^{\infty} \Pi_{T}(f-\varphi) \mathrm{e}^{j 2 \pi(f-\varphi) \Delta} \\
& \times \Pi_{T}(\varphi) \mathrm{e}^{j 2 \pi \varphi s T} \mathrm{e}^{-j 2 \pi \varphi \Delta} \mathrm{d} \varphi \\
K(f)= & \frac{1}{T^{2}} \int_{-\infty}^{\infty} \sum_{s=-\infty}^{\infty} \Pi_{T}(f-\phi) \\
& \times \Pi_{T}(\phi) \mathrm{e}^{-j 4 \pi \phi \Delta} \mathrm{e}^{j 4 \pi(f-\phi-(s / T)) \Delta} \\
& \times \Pi_{T}\left(f-\phi-\frac{s}{T}\right) \Pi_{T}\left(\phi+\frac{s}{T}\right) \mathrm{d} \phi .
\end{aligned}
$$

Noting again that the aforementioned expression is nonzero only when $s=0$, the expression simplifies to

$$
K(f)=\frac{1}{T^{2}} \int_{-\infty}^{\infty} \Pi_{T}^{2}(f-\phi) \mathrm{e}^{j 4 \pi(f-\phi) \Delta} \Pi_{T}^{2}(\phi) \mathrm{e}^{-j 4 \pi \phi \Delta} \mathrm{d} \phi
$$

$$
=\int_{-\infty}^{\infty} \Pi_{T}(f-\phi) \mathrm{e}^{j 4 \pi(f-\phi) \Delta} \Pi_{T}(\phi) \mathrm{e}^{-j 4 \pi \phi \Delta} \mathrm{d} \phi
$$

Expression (46) represents the convolution of a function with its conjugate. Hence, it can be reexpressed by rearranging the real and imaginary components as in

$$
\begin{aligned}
K(f)= & \int_{-\infty}^{\infty} \Pi_{T}(f-\phi) \cos (4 \pi(f-\phi) \Delta) \Pi_{T}(\phi) \\
& \times \cos (4 \pi \phi \Delta) \mathrm{d} \phi \\
& +\int_{-\infty}^{\infty} \Pi_{T}(f-\phi) \sin (4 \pi(f-\phi) \Delta) \Pi_{T}(\phi) \\
& \times \sin (4 \pi \phi \Delta) \mathrm{d} \phi .
\end{aligned}
$$

Using the linearity property of the integral operator and a trigonometric identity, the expression in (47) can be condensed

$$
K(f)=\Psi(4, f, \Delta)
$$

where the following function is defined:

$$
\Psi(x, f, \Delta)=\int_{-\infty}^{\infty} \Pi_{T}(f-\phi) \Pi_{T}(\phi) \cos (x \pi(f-2 \phi) \Delta) d \phi .
$$

3) Evaluating Term $\epsilon$ : Using the steps in the previous section, a preliminary expression for the Fourier transform of $\epsilon$ can be derived

$$
\begin{aligned}
E(f)=-\frac{2}{T} & \int_{-\infty}^{\infty} s\left(\frac{x+\Delta}{T}\right) s\left(\frac{x-\Delta}{T}\right) \mathrm{e}^{-j 2 \pi f x} \mathrm{~d} x \\
& \times \int_{-\infty}^{\infty} s\left(\frac{u+\Delta}{T}\right) s\left(\frac{u-\Delta}{T}\right) \mathrm{e}^{j 2 \pi f u} \mathrm{~d} u .
\end{aligned}
$$

Further manipulation yields

$$
\begin{aligned}
E(f)= & -\frac{2}{T} \int_{-\infty}^{\infty} \Pi_{T}(f-\phi) \mathrm{e}^{j 2 \pi(f-\phi) \Delta} \Pi_{T}(\phi) \mathrm{e}^{-j 2 \pi \phi \Delta} \mathrm{d} \phi \\
& \times \int_{-\infty}^{\infty} \Pi_{T}(f-\varphi) \mathrm{e}^{-j 2 \pi(f-\varphi) \Delta} \Pi_{T}(\varphi) \mathrm{e}^{j 2 \pi \varphi \Delta} \mathrm{d} \varphi
\end{aligned}
$$$$
E(f)=-\frac{2}{T} \Psi^{2}(2, f, \Delta)
$$

4) Evaluation of Terms $\chi$ and $\lambda$ : The Fourier transform of the expression obtained for $\chi$ is given by

$$
\begin{aligned}
X(f)= & \frac{1}{T} \sum_{s=-\infty}^{\infty} \int_{-\infty}^{\infty} s\left(\frac{x}{T}\right) s\left(\frac{x-s T}{T}\right) \mathrm{e}^{-j 2 \pi f x} \mathrm{~d} x \\
& \times \int_{-\infty}^{\infty} s\left(\frac{u-\Delta}{T}\right) s\left(\frac{u-s T+\Delta}{T}\right) \mathrm{e}^{j 2 \pi f u} \mathrm{~d} u .
\end{aligned}
$$


Further manipulations in the vein of those previously described yield the following:

$$
\begin{aligned}
X(f) & =\int_{-\infty}^{\infty} \Pi_{T}(f-\phi) \mathrm{e}^{j 2 \pi(f-\phi) \Delta} \Pi_{T}(\phi) \mathrm{e}^{-j 2 \pi \phi \Delta} \mathrm{d} \phi \\
& =\Psi(2, f, \Delta) .
\end{aligned}
$$

Because the function $\Psi(x, f)$ is symmetric with respect to frequency, it also holds that $X(f)=X_{\mathrm{TR}}(f)$. Evaluating $\lambda$ follows the exact same set of steps and results in

$$
\Lambda(f)+\Lambda_{\mathrm{TR}}(f)=-4 \operatorname{Tr}(f) \cdot \Psi(2, f, \Delta) .
$$

5) Evaluating $\psi(x, f)$ : It can be shown through Taylor series analysis that

$$
\Psi(x, f, \Delta)=\frac{T^{2}}{x \pi \Delta} \sin \left(\frac{x \pi \Delta}{T} \operatorname{Tr}(f)\right) .
$$

L'Hopital's rule yields that, for the case of $\Delta=0$, (56) simplifies to $T \cdot \operatorname{Tr}(f)$.

6) Final PSD Expressions: Combining the final expressions previously obtained yields expressions for the PSDs of the IM3 products and the error due to the interstage delay at the I channel baseband output. Recall that, to obtain the Q channel baseband output, it is only necessary to substitute $\Theta=\Theta+90^{\circ}$

$$
\begin{aligned}
S_{z z}(f)= & \frac{1}{4} A_{m}^{4} A_{\mathrm{CW}}^{2} T \cdot \operatorname{Tr}(f) \\
& \times\left[1-\operatorname{Tr}(f)\left(\frac{1}{2}+\frac{1}{2} \cos (2 \Theta)\right)\right] \\
S_{e e}(f)= & \frac{1}{16} A_{m}^{4} A_{\mathrm{CW}}^{2}\left[\Omega(f)+\Phi(f)\left(\frac{1}{2}+\frac{1}{2} \cos (2 \Theta)\right)\right] \\
\Omega(f)= & 6 T \cdot \operatorname{Tr}(f)+2 \Psi(4, f, \Delta)-8 \Psi(2, f, \Delta) .
\end{aligned}
$$

Further evaluation of (59) requires a Taylor series expansion of the $\Psi$ function. Retaining only the dominant terms yields

$$
\Omega(f) \approx \frac{16}{5 T^{3}} \pi^{4} \Delta^{4} \operatorname{Tr}^{5}(f) .
$$

Proceeding in the same manner yields

$\Phi(f)=-4 T \cdot \operatorname{Tr}^{2}(f)+8 \operatorname{Tr}(f) \Psi(2, f, \Delta)-\frac{4}{T} \Psi^{2}(2, f, \Delta)$.

Again, retaining only the dominant terms of the Taylor series expansion yields

$$
\Phi(f) \approx-\frac{16}{9 T^{3}} \pi^{4} \Delta^{4} \operatorname{Tr}^{6}(f) .
$$

The final result for the error PSD is then given by

$$
\begin{aligned}
S_{e e}(f) \approx A_{m}^{4} A_{\mathrm{CW}}^{2} & \frac{\pi^{4} \Delta^{4}}{T^{3}} \operatorname{Tr}^{5}(f) \\
& \times\left[\frac{1}{5}-\frac{1}{9} \operatorname{Tr}(f)\left(\frac{1}{2}+\frac{1}{2} \cos (2 \Theta)\right)\right] .
\end{aligned}
$$
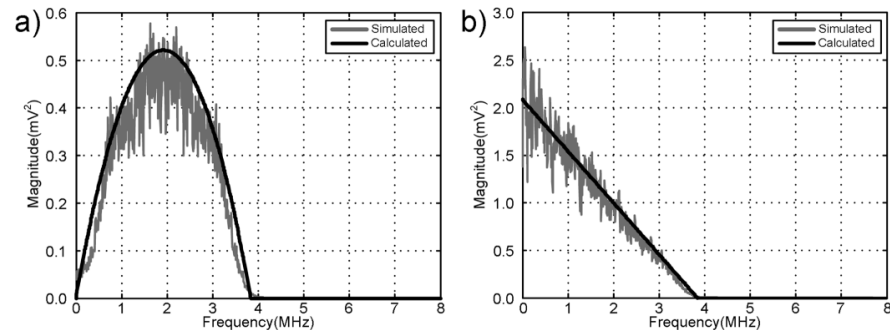

Fig. 13. Baseband PSD (single-sided) of squared QPSK modulated signal for (a) I and (b) $\mathrm{Q}$ channels when $Q=0^{\circ}$.
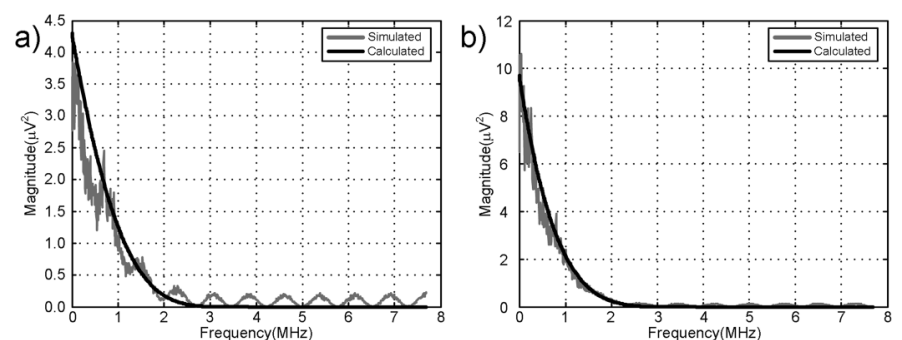

Fig. 14. Baseband PSD (single-sided) of squared QPSK modulated signal group delay-related error for (a) I and (b) $\mathrm{Q}$ channels when $Q=0^{\circ}$.

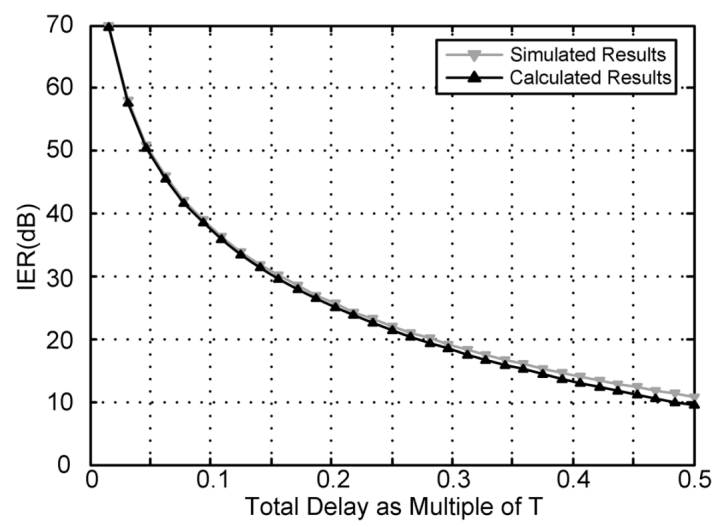

Fig. 15. Calculated IER as a function of group delay for $T=T R$.

The correctness of these calculations and the validity of the Taylor series truncation can be confirmed by considering Figs. 13 and 14. In this case, the results were obtained using a 641-tap sinc pulse-shaping channel filter in a discrete-time simulation construct where $F_{s}=245.76 \mathrm{MHz}$. These figures represent the case where $A_{m}=2, A_{\mathrm{CW}}=1$, and $\Delta=4$ ns.

Using these results, one can predict the IER due to this error mechanism. In Figs. 15 and 16, the "I" represents total IM3 power in order to provide a relevant gauge of total blocker power. "E" represents error quantities falling within the receiver (RX) bandwidth $1 / 2 T_{r}$, where $T_{r}$ is the chip rate of the received modulated signal. In Fig. 15, $\Delta$ is swept and $T=T_{r}=260.4 \mathrm{~ns}$, as in the UMTS specification test. In Fig. 16, the single-sided RX bandwidth and $\Delta$ are fixed at $1.92 \mathrm{MHz}$ and $4 \mathrm{~ns}$, respectively, while $T$ is varied. Note that, in the work presented in [9], $\Delta_{\mathrm{MAX}}$ was simulated at $0.5 \mathrm{~ns}$, 


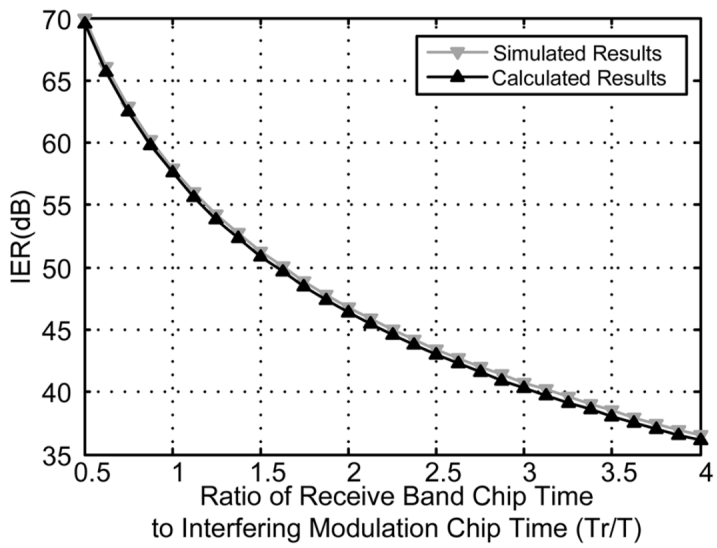

Fig. 16. Calculated IER versus blocker modulation chip rate for fixed delay.

and the alternate path IER target was $31 \mathrm{~dB}$ for a receiver with an uncorrected IIP3 of $-9 \mathrm{~dB}$. In this case, the effect due to the interstage group delay is negligible, as Fig. 15 shows.

\section{CONCLUSION}

In this paper, a multistage CMOS cubic-term generator has been evaluated for use in an IIP3-enhanced RF receiver using adaptive feedforward error cancellation. A proof is presented which shows that the interstage bandwidth of the multistage cubic-term generator can be restricted to pass only low beat-frequency IM2 terms while still producing the proper IM3 outputs. The SNR of the proposed architecture was shown to exceed system requirements for current levels that are much less than that of the receiver to be enhanced under worst case specification conditions. Finally, the effect of group delay between the nonlinear operations in the multistage cubic-term generator is evaluated using a time-domain statistical approach for a QPSK-modulated blocker, allowing a designer to choose an interstage delay appropriate to the modulation rates of the blockers encountered by the $\mathrm{RF}$ receiver.

\section{REFERENCES}

[1] M. Faulkner, "DC offset and IM2 removal in direct conversion receivers," Proc. Inst. Elect. Eng.-Commun., vol. 149, no. 3, pp. 179-184, Jun. 2002.

[2] V. H. Estrick and R. T. Siddoway, "Receiver distortion circuit and method," U.S. Patent 5 237 332, Aug. 17, 1993.

[3] M. Valkama, A. S. H. Ghadam, L. Antilla, and M. Renfors, "Advanced digital signal processing techniques for compensation of nonlinear distortion in wideband multicarrier radio receivers," IEEE Trans. Microw. Theory Tech., vol. 54, no. 6, pp. 2356-2366, Jun. 2006.

[4] L. Yu and M. Snelgrove, "A novel adaptive mismatch cancellation system for quadrature IF radio receivers," IEEE Trans. Circuits Syst. II, Analog Digit. Signal Process., vol. 46, no. 6, pp. 789-801, Jun. 1999.

[5] L. Yu and M. Snelgrove, "Signal processor for reducing undesirable signal content," U.S. Patent 6804 359, Oct. 12, 2004.

[6] V. Aparin, G. J. Ballantyne, C. J. Persico, and A. Cicalini, "An integrated LMS adaptive filter of TX leakage for CDMA receiver front ends," IEEE J. Solid-State Circuits, vol. 41, no. 5, pp. 1171-1182, May 2006.

[7] Z. S. Ebadi and R. Saleh, "Adaptive compensation of RF front-end nonidealities in direct conversion receivers," IEEE Trans. Circuits Syst. II, Exp. Briefs, vol. 55, no. 4, pp. 354-358, Apr., 2008.

[8] Y. Zheng, M. Cao, E. K. H. Teo, and H. K. Garg, "An adaptive filtering algorithm for direct-conversion receivers: Architecture and performance analysis," IEEE Trans. Circuits Syst. I, Reg. Papers, vol. 55, no. 4, pp. 1141-1148, May 2008.
[9] E. Keehr and A. Hajimiri, "Equalization of third-order intermodulation products in wideband direct conversion RF receivers," IEEE J. SolidState Circuits, vol. 43, no. 12, pp. 2853-2867, Dec. 2008.

[10] T. Nojima and N. Konno, "Cuber predistortion linearizer for relay equipment in $800 \mathrm{MHz}$ band land mobile telephone system," IEEE Trans. Veh. Technol., vol. VT-34, no. 4, pp. 169-177, Nov. 1985.

[11] N. Imai, T. Nojima, and T. Murase, "Novel linearizer using balanced circulators and its application to multilevel digital radio systems," IEEE Trans. Microw. Theory Tech., vol. 37, no. 8, pp. 1237-1243, Aug. 1989.

[12] W. Huang and R. E. Saad, "Novel third-order distortion generator with residual IM2 suppression capabilities," IEEE Trans. Microw. Theory Tech., vol. 46, no. 12, pp. 2372-2382, Dec. 1998.

[13] L. Roselli, V. Borgioni, V. Palassari, and F. Alimenti, "An active cuber circuit for power amplifier analog predistortion," in Proc. 33rd Eur. Microw. Conf., Oct. 2003, vol. 3, pp. 1219-1222.

[14] R. Sadhwani and B. Jalali, "Adaptive CMOS predistortion linearizer for fiber optic links," J. Lightw. Technol., vol. 21, no. 12, pp. 3180-3193, Dec. 2003.

[15] F. Shearer and L. MacEachern, "A precision CMOS analog cubing circuit," in Proc. IEEE NEWCAS, Jun. 2004, pp. 281-284.

[16] F. Shearer, "CMOS analog cubing circuits for radio-over-fiber predistortion," M.S. thesis, Carleton Univ., Ottawa, ON, Canada, 2005.

[17] T. Nesimoglu, C. N. Canagarajah, and J. P. McGeehan, "A broadband polynomial predistorter for reconfigurable radio," in Proc. Veh. Technol. Conf., May 2001, vol. 3, pp. 1968-1972.

[18] T. Rahkonen, O. Kursu, M. Riikola, J. Aikio, and T. Tuikkanen, "Performance of an integrated $2.1 \mathrm{GHz}$ analog predistorter," in Proc. Int. Workshop Integr. Nonlinear Microw. Millim.-Wave Circuits, Jan. 2006, pp. 34-37.

[19] E. Westesson and L. Sundstrom, "A complex polynomial predistorter chip in CMOS for baseband or IF linearization of RF power amplifiers," in Proc. IEEE ISCAS, Jun. 1999, vol. 1, pp. 206-209.

[20] T. S. Nielsen, S. Lindfors, S. Tawfik, and T. Larsen, "0.25 mm CMOS analog multiplier for polynomial predistorter," in Proc. Norchip Conf., Nov. 2004, pp. 191-194.

[21] H.-J. Song and C.-K. Kim, "An MOS four-quadrant analog multiplier using simple two-input squaring circuits with source followers," IEEE J. Solid-State Circuits, vol. 25, no. 3, pp. 841-848, Jun. 1990.

[22] J. Vuolevi, J. Manninen, and T. Pahkonen, "Cancelling the memory effects in RF power amplifiers," in Proc. IEEE ISCAS, May 2001, vol. 1, pp. 57-60.

[23] UE Radio Transmission and Reception (FDD), Tech. Specification Group, 3GPP, (TSG) RAN WG4, TS 25.101, Dec. 2007, v8.1.0.

[24] T. H. Lee, The Design of CMOS Radio-Frequency Integrated Circuits. Cambridge, U.K.: Cambridge Univ. Press, 2004, p. 184.

[25] P. J. Langlois and A. Demosthenous, "Sweet spots in moderate inversion for MOSFET squarer transconductors," IEEE Trans. Circuits Syst. II, Exp. Briefs, vol. 54, no. 6, pp. 479-483, Jun. 2007.

[26] K. Han, J. Gil, S.-S. Song, J. Han, H. Shin, C.-K. Kim, and K. Lee, "Complete high-frequency thermal noise modeling of short-channel MOSFETS and design of 5.2-GHz low noise amplifier," IEEE J. SolidState Circuits, vol. 40, no. 3, pp. 726-735, Mar. 2005.

[27] M. T. Yang, C. W. Kuo, P. P. C. Ho, D. C. W. Kuo, C. C. Chen, T. J. Yeh, C. Teng, J. Jayapalan, G. Brown, G. Yeap, Y. Du, and S. Liu, "CR018 wideband noise model for AMS/RF CMOS simulation," in Proc. IEEE RFIC Symp. Dig. Tech. Papers, Jun. 2007, pp. 643-646.

[28] V. Aparin and L. E. Larson, "Analysis and reduction of cross-modulation distortion in CDMA receivers," IEEE Trans. Microw. Theory Tech., vol. 51, no. 5, pp. 1591-1602, May 2003.

[29] A. V. Oppenheim, A. S. Willsky, and S. H. Nawab, Signals and Systems. Upper Saddle River, NJ: Prentice-Hall, 1997, p. 208.

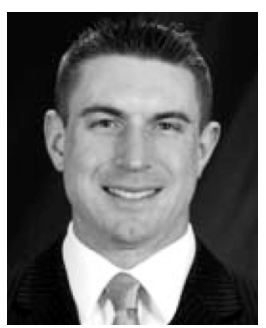

Edward A. Keehr (M'02-S'05) received the S.B. and M.Eng. degrees in electrical engineering from the Massachusetts Institute of Technology (MIT), Cambridge, in 2001 and 2002, respectively. Since 2005, he has been working toward the Ph.D. degree in electrical engineering in the California Institute of Technology (Caltech) High-Speed Integrated Circuits Group, Caltech, Pasadena.

From 1999 to 2002, as part of the MIT VI-A internship program, he held summer internships with Qualcomm, Inc., San Diego, CA, where he was a full-time Design Engineer specializing in analog and mixed-signal circuits from 2002 to 2005. His current research interests are RF transceiver circuits and architectures. 
Mr. Keehr is a member of Tau Beta Pi and Eta Kappa Nu. He was the recipient of a National Defense Science and Engineering Graduate Fellowship in 2005 and the Analog Devices Outstanding Student Designer Award in 2006.

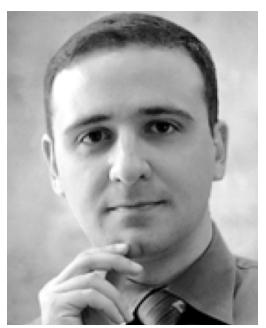

Ali Hajimiri (M'99) received the B.S. degree in electronics engineering from the Sharif University of Technology, Tehran, Iran, and the M.S. and Ph.D. degrees in electrical engineering from Stanford University, Stanford, CA, in 1996 and 1998, respectively.

He was a Design Engineer with Philips Semiconductors, where he worked on a BiCMOS chipset for Global System for Mobile Communication and cellular units from 1993 to 1994. In 1995, he was with Sun Microsystems, where he worked on the UltraSPARC microprocessor's cache RAM design methodology. During the summer of 1997, he was with Lucent Technologies (Bell Labs), Murray Hill, NJ, where he investigated low-phase-noise integrated oscillators. In 1998, he joined the faculty of the California Institute of Technology, Pasadena, where he is a Professor of Electrical Engineering and the Director of the Microelectronics Laboratory. He is a cofounder of Axiom Microdevices, Inc. He is the author of The Design of Low Noise Oscillators (Springer, 1999) and has authored and coau- thored more than 100 refereed journal and conference technical articles. He is the holder of more than 30 U.S. and European patents. His research interests are high-speed and RF integrated circuits.

Dr. Hajimiri is a member of the Technical Program Committee of the International Solid-State Circuits Conference (ISSCC). He has also served as an Associate Editor of the IEEE JOURNAL OF SOLID-STATE CIRCUITS (JSSC), an Associate Editor of the IEEE TRANSACTIONS ON CIRCUITS AND SYSTEMS: PART-II, a member of the Technical Program Committees of the International Conference on Computer-Aided Design, a Guest Editor of the IEEE TRANSACTIONS ON MiCROWAVE THEORY AND TECHNIQUES, and a member of the guest editorial board of the Transactions of the Institute of Electronics, Information and Communication Engineers of Japan. He is a Distinguished Lecturer of the IEEE Solid-State and Microwave Societies. He was selected to the top 100 innovators (TR100) list in 2004 and is a Fellow of the Okawa Foundation. He is the recipient of Caltech's Graduate Students Council Teaching and Mentoring Award as well as the Associated Students of Caltech Undergraduate Excellence in Teaching Award. He was the gold medal winner of the National Physics Competition and the bronze medal winner of the 21st International Physics Olympiad, Groningen, Netherlands. He was a corecipient of the IEEE JSSC Best Paper Award of 2004 and the ISSCC Jack Kilby Outstanding Paper Award, a two-time corecipient of the IEEE Custom Integrated Circuits Conference's Best Paper Awards, and a three-time winner of the IBM Faculty Partnership Award as well as the National Science Foundation CAREER Award. 